\title{
The evolutionary arms race between transposable elements and piRNAs in Drosophila melanogaster
}

\author{
Shiqi Luo ${ }^{1,2 \dagger}$, Hong Zhang ${ }^{1 \dagger}$, Yuange Duan ${ }^{1,3+}$, Xinmin Yao ${ }^{1}$, Andrew G. Clark ${ }^{4^{*}}$ and Jian Lu ${ }^{{ }^{*}}$
}

\begin{abstract}
Background: The piwi-interacting RNAs (piRNAs) are small non-coding RNAs that specifically repress transposable elements (TEs) in the germline of Drosophila. Despite our expanding understanding of TE:piRNA interaction, whether there is an evolutionary arms race between TEs and piRNAs was unclear.

Results: Here, we studied the population genomics of TEs and piRNAs in the worldwide strains of D. melanogaster. By conducting a correlation analysis between TE contents and the abundance of piRNAs from ovaries of representative strains of D. melanogaster, we find positive correlations between TEs and piRNAs in six TE families. Our simulations further highlight that TE activities and the strength of purifying selection against TEs are important factors shaping the interactions between TEs and piRNAs. Our studies also suggest that the de novo generation of piRNAs is an important mechanism to repress the newly invaded TEs.

Conclusions: Our results revealed the existence of an evolutionary arms race between the copy numbers of TEs and the abundance of antisense piRNAs at the population level. Although the interactions between TEs and piRNAs are complex and many factors should be considered to impact their interaction dynamics, our results suggest the emergence, repression specificity and strength of piRNAs on TEs should be considered in studying the landscapes of TE insertions in Drosophila. These results deepen our understanding of the interactions between piRNAs and TEs, and also provide novel insights into the nature of genomic conflicts of other forms.
\end{abstract}

Keywords: Transposable element, piRNA, Arms race, Co-evolution, Drosophila melanogaster

\section{Background}

The conflicts between two competing species could continuously impose selective pressures on each other, potentially causing an evolutionary arms race $[1,2]$. The "attackdefense" arms race, in which offensive adaptation in one species is countered by defensive adaptation in the other species (such as the predator-prey or the parasite-host asymmetry), could lead to three possible scenarios: 1) one side wins and drives the other to extinction, 2) one side reaches an optimum while displacing the other from its optimum; or, 3) the race may persist in an endless cycle

\footnotetext{
*Correspondence: ac347@cornell.edu; luj@pku.edu.cn

†'Shiqi Luo, Hong Zhang and Yuange Duan contributed equally to this work. ${ }^{4}$ Department of Molecular Biology and Genetics, Cornell University, Ithaca, NY 14853, USA

${ }^{1}$ State Key Laboratory of Protein and Plant Gene Research, Center for Bioinformatics, College of Life Sciences and Peking-Tsinghua Center for Life Sciences, Peking University, Beijing 100871, China

Full list of author information is available at the end of the article
}

[3]. Intra-genomic conflicts, the antagonistic interactions between DNA sequences (or their products) within the genome of the same species, can also lead to an evolutionary arms race at the molecular level [4-7]. Among various systems of genomic conflicts, an important form is the interaction between transposable elements (TEs) and the host genomes $[8,9]$. TEs are selfish genetic elements that are generally detrimental to the host organism [10-17]. The abundance of TEs varies dramatically across eukaryotes [10], ranging from 1\% [18] to more than $80 \%$ of the genome [19]. TEs impose a high fitness cost on the host organism through three possible mechanisms: 1) disrupting coding or regulatory regions of genes [20-24]; 2) eroding cellular energy and resources [25, 26]; or 3) nucleating ectopic recombination to induce chromosomal rearrangements [27-31].

Drosophila melanogaster provides a good system to study the molecular mechanisms and evolutionary dynamics of 
TEs [29, 32-35]. TEs make up at least 5\% of the euchromatic genome of D. melanogaster [36-41], and approximately $50-80 \%$ of mutations arising in D. melanogaster can be attributed to TE insertions [21, 42]. Although TE insertions in Drosophila have frequently been associated with adaptive evolution [43-47], TEs are overall selected against in Drosophila [20-30, 47-50]. PIWI-interacting RNAs (piRNAs), a class of small RNAs that specifically repress TEs expressed in animal germlines, were first discovered in Drosophila. The discovery of piRNAs has considerably deepened our understanding of the molecular mechanisms underlying the interactions between TEs and the host organisms [51-59]. The biogenesis and functional mechanisms of piRNAs exhibit features that are distinct from miRNAs and endogenous siRNAs [56, 60-67]. In Drosophila, piRNAs are small RNAs of approximately 23-29 nucleotides in length bound by Piwi-class Argonaute proteins (PIWI, AUB, and AGO3). Mature piRNAs are processed from piRNA precursors, which are usually transcribed from degenerated copies of TEs that form large clusters in heterochromatic regions of the Drosophila genome (called "piRNA clusters") [56, 68-76]. Mature piRNAs repress their target mRNAs through a positive feedback loop called the "Ping-Pong cycle", in which primary and secondary piRNAs alternatively cleave mRNAs of TEs [56, 77, 78].

The piRNA pathway well explains the molecular mechanisms underlying the $P-M$ system of hybrid dysgenesis in Drosophila [61, 79]. The P-element is a DNA transposon that invaded $D$. melanogaster from $D$. wilistoni by horizontal transfer within the last 100 years, and the $P$-element is still polymorphic in the populations of D. melanogaster [80-82]. Although P-elements replicate in a "cut-and-paste" manner, they increase their copy number in the genomes through homologous repair from sister strands $[83,84]$. Notably, many strains of $D$. melanogaster have generated piRNAs that specifically repress $P$-elements despite the recent insertions [61]. Since piRNAs are maternally deposited into the eggs and early embryos [56, 85-87], the maternal deposition of P-element corresponding piRNAs neatly explains the reciprocal cross difference in hybrid dysgenesis between $P$ and $M$ strains of $D$. melanogaster [61]. Besides, the piRNA machinery also provides novel insights into other longlasting evolutionary phenomena in Drosophila, such as the TE-repressing effects of the flamenco locus $[56,88]$, and the $I-R$ system of hybrid dysgenesis $[89,90]$.

Novel TE insertions are pervasive and highly variable in Drosophila. The host organisms could quickly develop novel piRNAs that specifically repress the novel invaded TEs through distinct mechanisms. For example, previous studies have demonstrated that the de novo production of piRNAs repressing $P$-elements could be achieved very rapidly in $D$. melanogaster after $P$-element invasions $[79,91-$ 93]. In addition, de novo piRNAs can also be generated in the flanking regions of novel inserted sites of other TE families [71, 94-96]. Besides being generated from de novo sites, piRNAs can also be produced from the preexisting piRNA clusters after a novel TE invades into that cluster. For example, in $D$. simulans, piRNAs were quickly produced to suppress the $P$-elements that were inserted into pre-existing piRNA clusters [97]. Also, after introducing the Penelope TE into D. melanogaster, piRNAs were generated to suppress Penelope after this TE jumped into a pre-existing piRNA cluster [98]. Nevertheless, it yet remains unclear which of the two mechanisms is the dominant mechanism to produce novel piRNAs that suppress a novel invading TE.

Given the importance of piRNAs in repressing TEs, several groups have studied the evolutionary dynamics of TE/piRNA interactions using Drosophila as the model [95, 99-101]. Previously, we (Lu \& Clark) modeled the population dynamics of piRNAs and TEs in a population genetics framework [99]. Our results suggest that piRNAs can significantly reduce the fitness cost of TEs, and that TE insertions that generate piRNAs are favored by natural selection [99]. Similar conclusions were drawn by other studies as well $[102,103]$. Since piRNAs suppress activities of the target TEs, one might intuitively expect to observe a negative correlation between the copy numbers/activities of TEs and piRNAs at the population level. However, other studies have shown that there might be evolutionary arms race between TEs and TE-derived piRNAs from different aspects. First, TEderived piRNA abundance tends to be positively correlated with TE expression in individual strains of $D$. melanogaster and D. simulans [101, 104]. Second, it was shown that although the signal of ping-pong amplification and piRNA cluster representation affect TE-derived piRNA abundance in a strain, the level of piRNA targeting is rapidly lost for inactive TEs in that strain [101]. Third, TE expression is negatively correlated with activities of piRNA pathway genes at the population level [104], and intriguingly, the effector proteins in piRNA machinery also show strong signatures of adaptive evolution [105-107]. These results suggest that the genes in the piRNA pathway machinery might be involved in the arms-race co-evolutionary processes between TEs and piRNAs (or the host organisms). Moreover, our previous studies also demonstrated that piRNAs may provide a shelter for TEs in the genomes since the detrimental effects of TEs are alleviated [99]. Based on these observations, here, we hypothesized the competitive interactions between TEs and piRNAs could lead to an arms race because of the detrimental effects imposed by TEs and the selective advantage conferred by piRNAs in repressing TEs. Previously, Song et al. sequenced small RNAs in ovaries of $16 \mathrm{D}$. melanogaster strains from the DGRP project $[108,109]$. However, they did not find a simple 
linear correlation between the global piRNA expression and novel TE insertions (the polymorphic insertions) across the 16 DGRP strains [95]. Here, we aimed to test the TE/piRNA evolutionary arms race hypothesis with another population genomic dataset of $D$. melanogaster. Under the piRNA:TE evolutionary arms race scenario, we expect to observe a positive correlation between TE content and piRNA abundance among different strains.

In this study, we first examined the abundance of TEs and their respective piRNAs in the worldwide Global Diversity Lines (GDL) of $D$. melanogaster [110]. We found the novel TE insertions frequently induced de novo piRNA generation from the flanking regions of the insertion sites. We then conducted correlation analysis between TE contents and the abundance of piRNAs from ovaries of 26 representative strains of $D$. melanogaster, and detected significantly positive correlations for six TE families. We also conducted forward simulations with the parameters optimized for $D$. melanogaster to investigate the factors influencing the evolutionary arms race between TEs and piRNAs.

\section{Results and discussion}

The contents of TEs vary across populations of $D$.

\section{melanogaster}

Empirical tabulation of the abundances of TEs and piRNAs across a series of wild-derived fly strains will serve as the initial substrate for learning about their coevolutionary dynamics. The strains of $D$. melanogaster sequenced in the GDL project were collected from five continents (B, Beijing; N, Netherlands; I, Ithaca, New York; T, Tasmania; and Z, Zimbabwe), and these strains were sequenced at $\sim 12.5 \times$ coverage [110]. For each of the 81 strains sequenced with the Illumina $100 \mathrm{bp}$ paired-end protocol, we mapped the genomic shotgun reads to the reference genome of D. melanogaster and characterized TE insertions with two complementary methods (Methods). First, for each TE insertion annotated in the reference genomes of $D$. melanogaster (called the "known" insertions), we examined whether it was present in the 81 GDL strains based on the mapping results of the flanking sequences. Among the 3544 known TE insertions that have unique boundary sequences in the reference genome, the average copy number $( \pm$ s.e.) in each strain ranged from $1204.3 \pm 8.4$ to $1309.1 \pm 3.5$ in the five populations (Fig. 1a). Notably, 600 (26.8\%) of the known TE insertions were not found in any GDL strain, supporting the notion that unique transposon insertions are pervasive in the populations of D. melanogaster [100]. As expected [31], these reference-genome-specific insertions are mainly caused by longer TEs (the length is $5088.9 \pm 131.1$ versus $1853.1 \pm 52.0 \mathrm{nts}$ of the remaining TEs in the reference genome; $P<10^{-10}$, Kolmogorov-Smirnov test [KS test]). Second, in each GDL strain, we employed TEMP [111], which was designed to detect novel TE insertions in Drosophila, to systematically identify possible novel TE insertions that are not present in the reference genome of $D$. melanogaster, and we further filtered the original TEMP results based on strict criteria to remove possible falsepositive results (Methods). In total, we identified 11,909 novel insertion sites of TEs that were present in the GDL strains but absent in the reference genome, and the average number of novel insertions in each strain ranges from 171 to 388 in the five populations (Fig. 1b). To assess the TEMP performance in TE detection, we compared the results obtained in the $\sim 12.5 \times$ coverage of ZW155 strain versus those obtained with an independent $100 \times$ coverage paired-end re-sequencing of this same strain [110]. Of the 238 novel insertions detected in the $12.5 \times$ sequencing, 198 were independently verified using the $100 \times$ coverage resequencing result, yielding a call rate repeatability of $83.2 \%$. Among the novel insertions, $61.3 \%$ of the insertions were caused by LTRs, $19.2 \%$ caused by DNA transposons and $14.6 \%$ mediated by non-LTRs.

As previously shown $[112,113]$, the novel TE insertion sites are significantly enriched in the $\mathrm{X}$ chromosome after controlling for the size differences of chromosomes (Table 1, Fig. 1c). The majority of the novel insertions occurred in introns (56.9\%), followed by 3' UTRs (5.60\%), ncRNAs (3.98\%), 5' UTRs (2.37\%), and CDSs (1.80\%) (Additional file 1: Table S1). TE insertions often disrupt CDSs or regulatory sequences $[31,40,46]$. To explore the impact of TE insertions on the expression levels of the host genes, we examined the whole-body transcriptomes of adult females for 5 GDL strains (B12, I17, N10, T05, and ZW155) [114]. As expected $[50,95,115]$, we found genes with novel TE insertions in exons, especially in CDSs, had significantly reduced expression levels (Fig. 1d) when we compared gene expression levels in the strains with a TE insertion versus the strains without that particular TE insertion. By contrast, TE insertions in introns or $500 \mathrm{bp}$ upstream of the TSS (transcriptional start site) are not associated with significant changes in gene expression levels (Fig. 1d).

To identify the adaptive TE insertion events that left footprints in the genomes, we calculated Tajima's $D$ [116] and Fay \& Wu's $H$ [117] values in a binned window of $10 \mathrm{~kb}$ (Additional file 1: Figures S1 and S2) and the composite likelihood ratio (CLR) [118-120] with SweeD [121] in each local and the global population (Additional file 1: Figure S3). We identified 24 highfrequency TE insertions (present in at least 5 strains) that have flanking SNPs with $D<-1$ and $H<-1$ in the local or global populations (Additional file 1: Table S2), among which three TE insertions fall within the top 5\% CLR distribution in the corresponding analysis, including one 412 insertion in Dystrophin (Additional file 1: Figure S4). These results suggest such TE insertions potentially lead to local adaption in the GDL strains. 


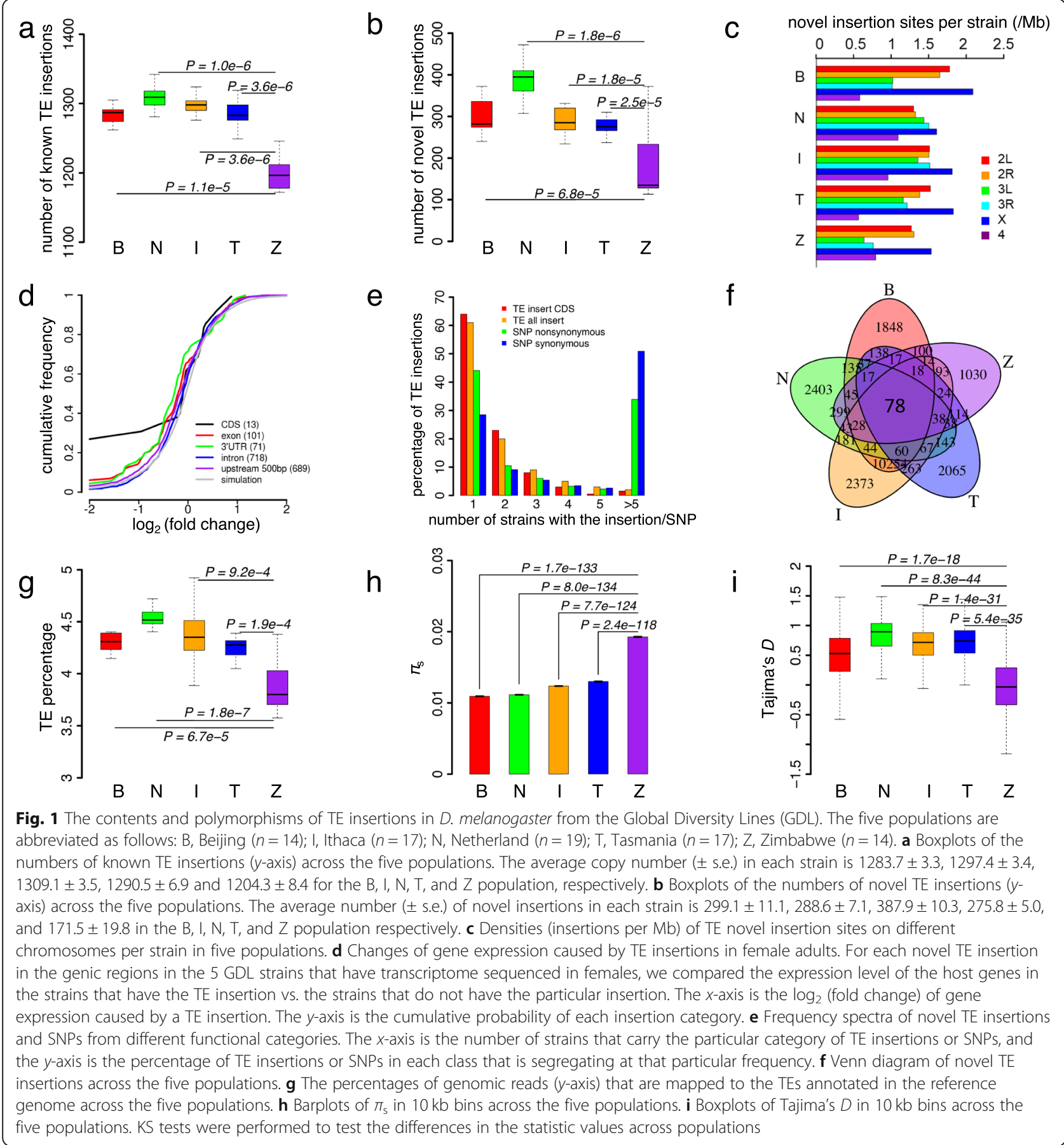

Compared to the derived synonymous or nonsynonymous mutations (Methods), the frequency spectra of the TE insertions are significantly skewed to lower frequencies $(P<0.0001$ in each comparison, Fisher's exact tests; Fig. 1e), suggesting that novel insertions of TEs are overall under stronger purifying selection. Specifically, among the novel insertions of TEs, 9719 (61.9\%) were detected in a single GDL strain, 537 (4.51\%) were present in more than five strains, and only 78 insertions were shared among all the five populations (Fig. 1f). Accordingly, the multidimensional scaling (MDS) analysis of the known (Additional file 1: Figure S5a) and novel (Additional file 1: Figure S5b) insertions of TEs suggests that strains from the same population are well clustered. Interestingly, the $\mathrm{Z}$ strains, in general, have the lowest numbers of known (Fig. 1a) and novel (Fig. 1b) TE insertions. Moreover, the $\mathrm{Z}$ strains have significantly lower fractions of reads from TEs that are mapped on the 
Table 1 Summary of the novel TE insertions in different chromosomes in the GDL strains

\begin{tabular}{|c|c|c|c|c|c|c|c|c|c|}
\hline \multicolumn{2}{|c|}{ Chromosome } & \multicolumn{2}{|c|}{ All novel insertions } & \multicolumn{3}{|c|}{ Frequency } & \multicolumn{3}{|c|}{ Frequency (TE > 5 kb) } \\
\hline Name & Length & Observed & Expected & 1 & $2-5$ & $>5$ & 1 & $2-5$ & $>5$ \\
\hline $2 \mathrm{~L}$ & $23,513,712$ & 2279 & 2088.09 & 1486 & 717 & 76 & 1047 & 444 & 20 \\
\hline $2 \mathrm{R}$ & $25,286,936$ & 2328 & 2245.55 & 1474 & 741 & 113 & 1060 & 447 & 53 \\
\hline $3 L$ & $28,110,227$ & 2013 & 2496.27 & 1142 & 753 & 118 & 837 & 471 & 52 \\
\hline $3 R$ & $32,079,331$ & 2352 & 2848.74 & 1187 & 1009 & 156 & 884 & 652 & 69 \\
\hline 4 & $1,348,131$ & 73 & 119.72 & 55 & 16 & 2 & 40 & 10 & 1 \\
\hline$x$ & $23,542,271$ & 2844 & 2090.62 & 1996 & 780 & 68 & 1374 & 442 & 30 \\
\hline
\end{tabular}

reference genome than the other four populations $(P<$ 0.0001 in each comparison, KS test, Fig. $1 \mathrm{~g}$ ). Since some TEs are absent in the reference genome of $D$. melanogaster [122] and the level of TE sequence diversity might be different in the five populations, we also mapped the genomic reads on the TE sequences annotated in Drosophila Genome Project (BDGP) TE dataset and RepBase Update [123] using BLAT [124] with different thresholds of mapping length and identity. We still obtained similar results despite the different mapping thresholds (Additional file 1: Figure S6). Previous studies indicate the $\mathrm{Z}$ population, which has a larger effective population size than the non-African populations [125-129], experienced a recent growth [130-132], and the non-African populations often experienced bottleneck after migration out of Africa [130, 132]. Consistently, the Z population in the GDL strains have significantly higher nucleotide diversity $\left(\pi_{\mathrm{s}}\right)$ and lower Tajima's $D$ values than the $\mathrm{N}, \mathrm{I}$, $\mathrm{B}$, and $\mathrm{T}$ populations $\left(P<10^{-16}\right.$ in each comparison, $\mathrm{KS}$ tests; Fig. 1h, i). Since the efficacy of natural selection is inversely influenced by the effective population size [133], purifying selection might have eliminated deleterious TE insertions more efficiently in the $\mathrm{Z}$ strains.

Altogether, in this study, we detected abundant TE insertions that are polymorphic in the population of $D$. melanogaster, and the $\mathrm{Z}$ population from Africa harbors fewer TE insertions than other populations, which might be related to the stronger purifying selection. The heterogeneity of TE insertions among strains of D. melanogaster enables us to test the possible evolutionary arms race between TEs and their suppressors at the population level.

\section{Profiling piRNAs in ovaries of 10 representative GDL strains by deep sequencing}

To explore the impact of piRNA repression on the TE distributions in the GDL strains, we deep-sequenced small RNAs from ovaries of 3-5-day-old females in 10 representative GDL strains that were collected from five continents (see Additional file 1: Table S3 for sequencing statistics). We mapped the small RNAs onto the reference genome of $D$. melanogaster and TE sequences collected from BDGP TE dataset and RepBase Update
[123] (Methods). In case a small RNA read was mapped to multiple locations, it was equally split across these locations. After removing reads that mapped to rRNAs, tRNAs, miscRNAs, ncRNAs and miRNAs, the remaining small RNAs that mapped to the reference genome show a major peak at $25 \mathrm{nt}$ (ranging from 23 to $29 \mathrm{nts}$ ) and a minor peak at $21 \mathrm{nt}$ (ranging from 20 to $22 \mathrm{nts}$ ), which are typical lengths of piRNAs and endogenous siRNAs, respectively (Fig. 2a). As expected [56, 86, 111, 134, 135], $\sim 72.1 \%$ of the piRNA-like reads (23-29 nt) in our study had uridine in the first position of the $5^{\prime}$-end (referred as "1 U", Fig. 2b). Overall, $45.6-51.7 \%$ of all the mapped 23-29 nt piRNA-like reads were from TEs, suggesting TEs are the major source for piRNAs. Although 34.8$39.7 \%$ of all the mapped piRNA-like reads were located in previously identified piRNA clusters [56, 86, 134, 135], 26.0-31.8\% of them mapped onto TEs outside the known clusters (Fig. 2c). If we only considered the piRNA-like reads that were uniquely mapped to the genome and TE reference sequences, we found 25.8-43.6\% of the piRNA reads were mapped to the known piRNA clusters, and $3.7-9.2 \%$ of them were mapped to TEs outside the piRNA clusters (Fig. 2d). These results suggest some piRNAs are either produced from novel piRNA clusters or through a piRNA-cluster-independent approach. In the "Ping-Pong" cycle of piRNA suppression and amplification, a sense-strand piRNA that is bound by Ago3 recognizes a complementary piRNA transcript and Ago3 cleaves the target at the site corresponding to the 10th nucleotide of the loaded piRNA, generating a new antisense piRNA that is bound by Aub. Then the Aub-loaded piRNA recognizes and cleaves a complementary TE transcript, generating a new piRNA identical to the initial Ago3-loaded piRNA [56, 78, 86, 134, 135]. The $10 \mathrm{nt}$ overlap between an Ago3-loaded sense piRNA and Aub-loaded antisense piRNA is a hallmark for piRNA biogenesis and functioning in the presence of the active target TE. In each sample, we detected significant "Ping-Pong" signals in all the piRNA-like reads (Fig. 2e), highlighting that our sequencing results have well captured the interactions between piRNAs and active TEs. 


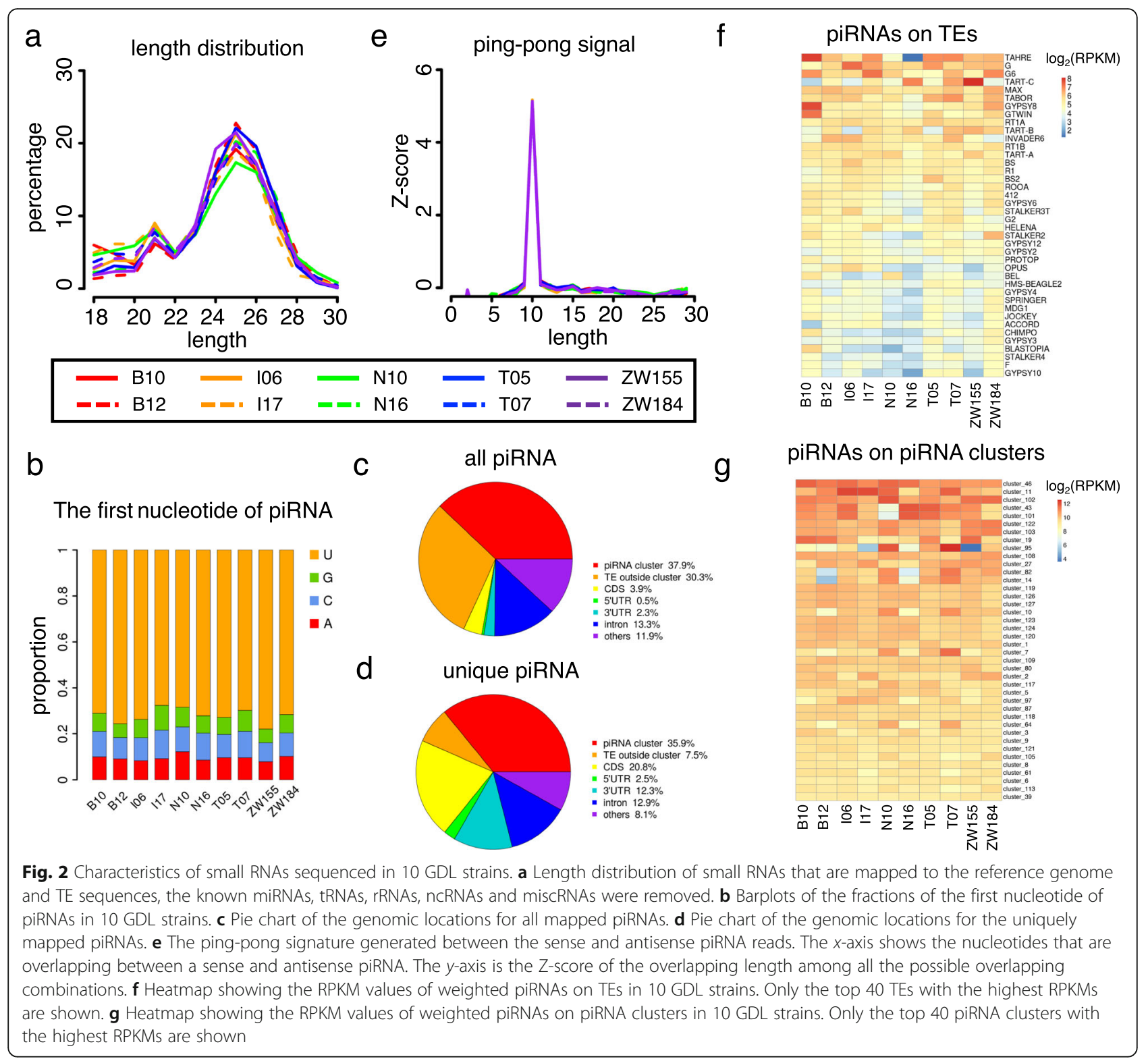

Among various TE families, the reference sequences of TAHRE, G, G6, TART-C, and MAX have the highest density of piRNAs (Fig. 2f). For the $29 \mathrm{TE}$ families whose reference sequences have the mean piRNAs density $>20$ RPKM among strains, the median coefficients of variation ( $c v$, defined as sd/mean of expression across strains) is 0.38 , with piRNAs on the sequences of TARTC, GYPSY8, GTWIN, OPUS and BEL families most variable across the 10 GDL strains. For the 56 known piRNA clusters that have piRNA density $>20$ RPKM, the $c v$ value ranged from 0.054 to 0.74 , with a median value of 0.20 , suggesting the piRNAs generated in these clusters are also variable across strains (Fig. $2 \mathrm{~g}$ ).

Besides being generated from de novo sites, piRNAs can also be produced from the pre-existing piRNA clusters after a novel TE invades into that cluster (Fig. 3a). However, it yet remains unclear which of the two mechanisms is the dominant mechanism to produce novel piRNAs that suppress a novel invading TE. We found 18 novel TE insertions in the known piRNA clusters in the $10 \mathrm{GDL}$ strains. For example, the X-linked flamenco piRNA cluster harbors the largest number of novel TE insertions in the $10 \mathrm{GDL}$ strains (Five novel TE insertions regions were observed in this locus, Additional file 1: Figure S7), followed by the piRNA cluster $42 A B$ on $2 \mathrm{R}$, which hosts three novel TE insertions (Additional file 1: Figure S8). By contrast, we found 343 out of 2632 (13.0\%) novel TE insertions that have signals of de novo 23-29 nt piRNAs in at least one strain with the uniquely mapped reads (Table 2 ). Consistent with 


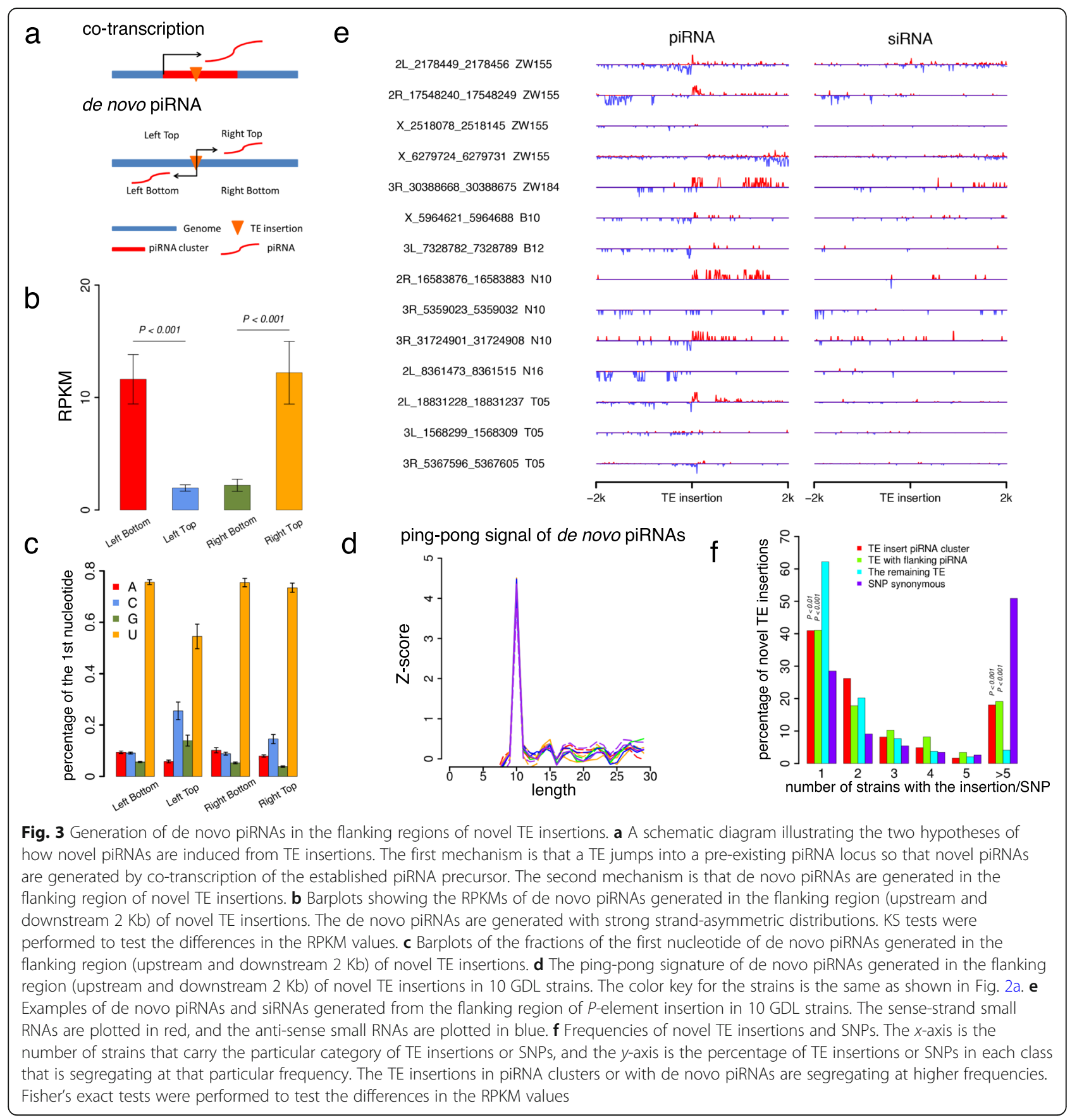

previous observations [94, 95], the de novo piRNAs are generated with strong strand-asymmetric distributions: the majority of the piRNAs in the left flank are in the antisense strands while most of the piRNAs in the right flank are generated in the sense strands (Fig. $3 \mathrm{~b}$ and Additional file 1: Figure S9). The piRNAs in the flanking regions are also enriched in $1 \mathrm{U}$ signatures (Fig. 3c) and show the typical ping-pong signature (Fig. 3d). Notably, we frequently detected endogenous siRNAs in those regions flanking the TE insertion (Additional file 1: Figure S10, an example of
P-element is displayed in Fig. 3e), although it is yet unclear whether such siRNAs are involved in the induction of the de novo piRNAs.

Our previous results suggest that novel insertions in the piRNA clusters are favored by natural selection, since they generate piRNAs that repress active TEs [99]. Accordingly, in the GDL strains the novel insertions in the piRNA clusters are overall segregating at higher frequencies than the remaining novel insertions (Fig. 3f). Interestingly, the TE insertions that have de novo piRNA 
Table 2 Novel TE insertions in the 10 strains that have piRNAs (23-29 nt) uniquely mapped to the regions $2 \mathrm{~kb}$ up- or downstream of the inserted sites

\begin{tabular}{lll}
\hline Strain & Novel TE insertion regions & $\begin{array}{l}\text { Novel TE insertion regions } \\
\text { with unique piRNAs }\end{array}$ \\
\hline B10 & 336 & $54(16.1 \%)$ \\
B12 & 292 & $39(13.4 \%)$ \\
106 & 260 & $22(8.5 \%)$ \\
I17 & 255 & $26(10.2 \%)$ \\
N10 & 455 & $33(7.3 \%)$ \\
N16 & 306 & $42(13.7 \%)$ \\
T05 & 251 & $31(12.4 \%)$ \\
T07 & 272 & $24(8.8 \%)$ \\
ZW155 & 248 & $42(16.9 \%)$ \\
ZW184 & 370 & $49(13.2 \%)$ \\
\hline
\end{tabular}

production signals in the flanking regions are also segregating at higher frequencies than the remaining TE insertions (22.6 and $6.17 \%$ of the TE insertions are segregating in at least 5 strains for the former and latter classes, respectively; $P<0.001$, Fisher's exact test; Fig. 3f). It is possible that these novel insertions might be advantageous, since the de novo piRNAs might repress other detrimental TEs through trans-acting effects. Nevertheless, we could not exclude the possibility that the de novo piRNAs generated by a novel insertion will alleviate the deleterious effects of the inserted TE itself so that it is under relaxed selective constraints.

Together, our results suggest that de novo induction is more prevalent than piRNA cluster trapping for novel piRNA biogenesis in natural populations of D. melanogaster. As expected, novel TE insertions with piRNA cluster trapping and de novo piRNA generation tend to segregate at higher frequencies in the populations. Importantly, the abundance of piRNAs is variable in the ovaries of different D. melanogaster strains, raising the possibility that the variation in piRNAs might be coupled to the variation in TEs.

\section{Relationship between piRNA abundances and TE copy numbers across strains of $D$. melanogaster}

To test the evolutionary arms race between piRNAs and TEs at the population level, we examined the relationship between piRNA abundances and the total TE copy numbers across the 10 representative GDL strains of $D$. melanogaster. In each strain, we predicted the target TEs of the piRNAs by requiring the perfect match between the 2-11 positions of piRNAs and the target sequences (Methods). For a reference TE sequence, we calculated the density of piRNAs that putatively target that TE. In case a piRNA targets multiple TE reference sequences, it was equally split and assigned to all the predicted targets (Methods). Notably, the length of a TE is significantly positively correlated with the weighted abundance of piRNAs targeting that TE (Additional file 1: Figure S11), suggesting longer TEs which are in general more deleterious [31] are also more likely targeted by piRNAs. Across the $10 \mathrm{GDL}$ strains of D. melanogaster, only P-element out of the 105 tested TE families showed a significantly positive Spearman's correlation between TE DNA copy numbers and the weighted abundances of antisense piRNAs after multiple testing correction (adjusted $P<0.05$ was used as cutoffs; Additional file 2: Table S4).

A previous study [95] has sequenced small RNAs in ovaries of $16 \mathrm{D}$. melanogaster strains from the DGRP project $[108,109]$. Similar to our results with the 10 GDL strains, that study also did not detect significant correlations between TE insertions and piRNAs in 16 D. melanogaster strains after correcting for multiple testing [95]. To increase the statistical power of the correlation analysis, we combined the data from both sources and conducted the correlation analyses. The correlations between TE DNA copy numbers and antisense piRNA densities tended to mixed across the 26 strains of $D$. melanogaster (the Spearman's Rho value was positive for 65 families and negative for 40 families, Additional file 2: Table S4). Of note, we did not observe significant differences in Rho values among DNA transposons, LTR, and non-LTR TE families (Fig. 4a). However, we found significantly positive Spearman's correlations (adjusted $P<0.05$ ) between TEs and antisense piRNAs for six TE families, among which five were retrotransposons (CHOUTO is LTR, and BAGGINS, TAHER, TART-B, TART-C are non-LTRs), and Pelement was DNA transposon (Fig. 4b). Thus, increasing the sample size in future studies will deepen our understanding of the evolutionary arms race between TEs and piRNAs at the population level.

The complete $P$-element (2907 bp in length) encodes a functional transposase and is autonomous. However, most TE sequences from the P-element family are internally deleted and are non-autonomous [136]. Accordingly, our genome alignments of the shotgun Illumina reads revealed more reads that mapped to the ends of the complete $P$-element, suggesting the widespread existence of the defective $P$-element in the GDL strains (Fig. 4c). By contrast, only a small fraction of the $P$ element fragments is full-length (Fig. 4c). We detected the $P$-element insertions in all five populations, with the median insertion number of $13.5,12,21,13$, and 10 for the $\mathrm{B}, \mathrm{I}, \mathrm{N}, \mathrm{T}$, and $\mathrm{Z}$ population, respectively. In total, we detected 133 insertions of P-element in these 10 GDL strains, and found de novo piRNAs flanking the $P$ element for 14 of these insertions (Fig. 3e). The $P$-element-derived piRNAs were mainly located in the $5^{\prime}$ and $3^{\prime}$ ends of P-element and their abundance varied dramatically across the 10 GDL strains (Fig. 4c). The copy number of the active part (position 819-2527) of the 


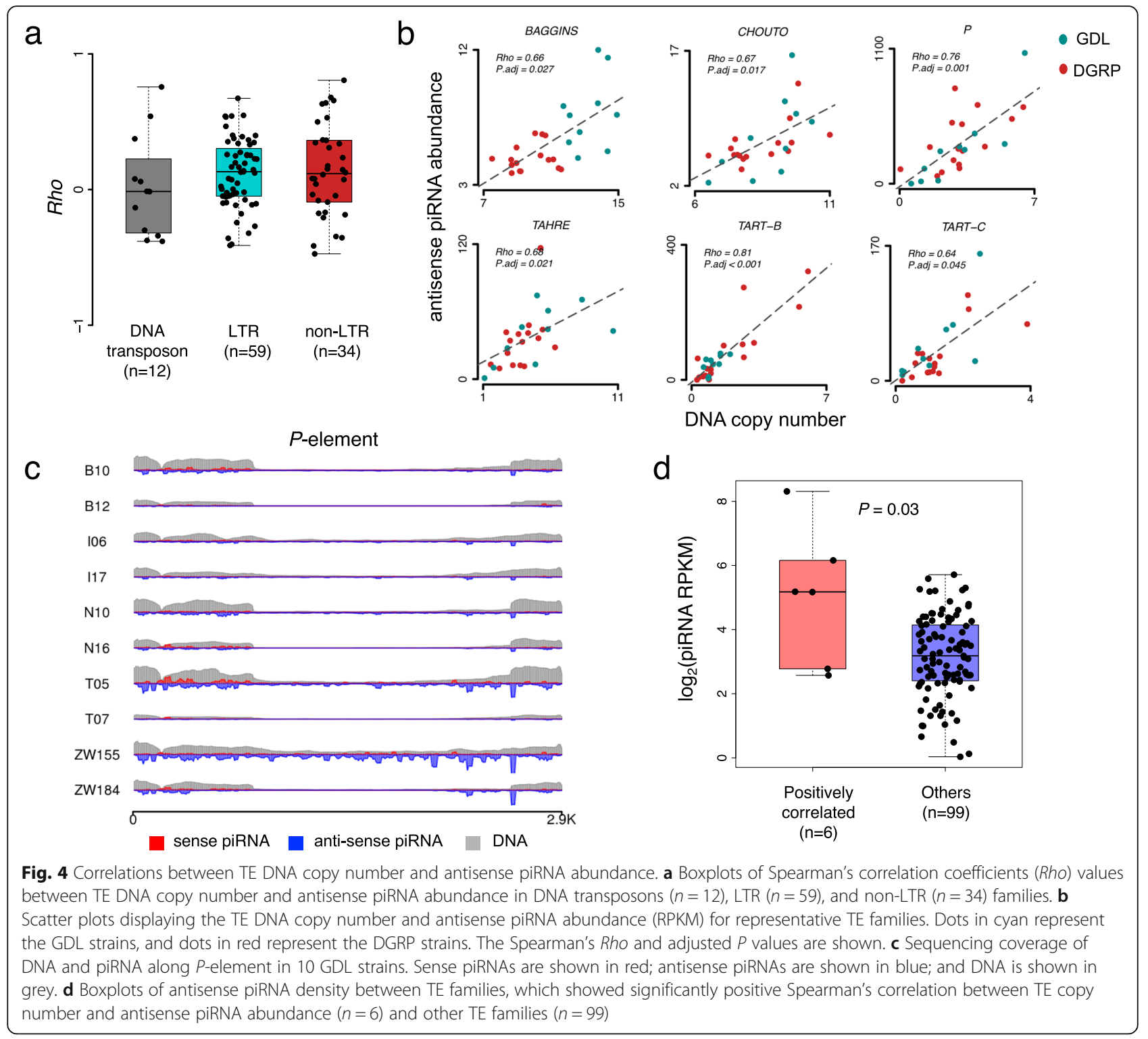

full-length $P$-element was significantly positively correlated with the abundance of antisense piRNAs in ovaries of the 26 strains of D. melanogaster (Spearman's Rho = $0.76, P=1.41 \times 10^{-3}$ in the correlation analysis; Fig. $4 \mathrm{~b}$ ). These results suggest the existence of an evolutionary arms race between $P$-elements and piRNAs in the populations of D. melanogaster.

There are two different piRNA pathways in the germline and somatic cells of the gonads of Drosophila [86, 137]. In the somatic ovarian follicle cells, the piRNAs from flamenco locus are loaded on Piwi and mainly target TEs from the gypsy family, while the Ago3-dependent PingPong cycle primarily occurs in the germline. Based on the Ping-Pong signals and Piwi-binding patterns, TEs were classified as germline-specific, somatic and intermediate groups $[86,137]$. Among the six TE families that show positive correlations between TE DNA copy numbers and antisense piRNA densities, BAGGINS, TART-B, TART-C, and TAHER belong to the germline-specific group in which piRNAs showed salient ping-pong signals. Moreover, we also found TEs of the six families overall have a significantly higher density of antisense piRNAs than the remaining 99 TE families ( $P=0.03$, Fig. $4 \mathrm{~d})$, affirming the thesis that the observed evolutionary arms race is caused by the tight interaction between TEs and piRNAs.

Altogether, here we combined data from two sources and detected significantly positive Spearman's correlations between TEs and antisense piRNAs for six TE families. For the remaining TE families that we did not detect statistically significant correlations, it is possible that the limited dataset (26 strains were used) or our methods lacked the power in detecting the true signals, and this does not 
necessarily suggest that evolutionary arms race does not exist in those TE families. TEs of different families often vary in many aspects, such as the preferences of insertion sites, the invasion history, and replication rates [113, 138], all of which might affect the relationships between TE and piRNA abundances. Therefore, more factors and more complex (or specific) models need to be considered in studying the arms race between TEs and piRNAs.

\section{The model of TE:piRNA interactions}

In order to explore how the observations of variation in TE and piRNA abundances may impact their coevolution, we conducted forward simulations of TE:piRNA interaction dynamics in populations of $D$. melanogaster using procedures similar to those we described previously [99]. Briefly, we assumed: 1) a diploid, panmictic, constantsized (effective population size $N_{e}$ ) Wright-Fisher population (non-overlapping generations); 2) the chromosome size is $100 \mathrm{Mb}$ and the homogeneous recombination rate per nucleotide is $r$; 3) in each generation the probability that a TE inserts into a new site and becomes a piRNAgenerating site is $f, 4$ ) the duplication rate of a TE or piRNA locus per generation is $d ; 5)$ the probability that a TE is excised or inactivated is $i$; 6 ) the probability that a TE mutates to a new subtype and escapes the repression effect of a piRNA is $e$; and 7) only the TE that does not generate piRNAs can replicate; a TE of subtype $j$ that is not targeted by any matching piRNA replicates at rate $u$ per element per generation; and a TE of $x_{j}$ sites that is targeted by the matched piRNAs with $y_{j}$ sites replicates at a rate $u /\left(1+R \cdot \frac{y_{j}}{x_{j}}\right)$, where $R$ is a constant representing piRNA repression efficiency. Note that in our model TEs and piRNA loci are on the same scale, piRNAs repress TEs with "enzymatic" kinetics and in a dosage-dependent manner, and the activities of TEs in each individual are determined by the abundance of matched piRNAs as well as the numbers of TEs which compete with each other for the matched piRNAs in that individual. We also considered sequence divergence between TE copies, and the piRNAs only repress TEs of the same subtype. We assumed TEs overall imposed fitness cost in a negative epistatic manner $[99,139,140]$. Specifically, the fitness of each individual in each generation is modeled by an exponential quadratic function, $w=e^{-s \cdot a \cdot n-\frac{1}{2} s \cdot b \cdot n^{2}+p \cdot\left(-s \cdot a \cdot m-\frac{1}{2} s \cdot b \cdot m^{2}\right)}$, where $a$ and $b$ are constants, $s$ is a scaling constant, $n$ is the effective number of active TEs, with $n=\sum_{j=1}^{k} x_{j} /\left(1+R \cdot y_{j} / x_{j}\right.$ ) and $x_{j}$ and $y_{j}$ being the copy numbers of TE and piRNA sites for a TE subtype $j$ in that individual; $m$ is the number of excessive piRNAs, with $m=\max \left(0, \sum_{j=1}^{k} y_{j}-\mathrm{x}_{j}\right)$, and $p$ is the penalty coefficient of excessive piRNAs on the fitness of the host organism. Note here we assumed excessive dosage of piRNAs might cause off-target effects on the normal transcriptomes and hence reduce the fitness of the host organism [107]. Moreover, although our model is designed for the "copy-and-paste" replication of retrotransposons, it is also applicable to DNA transposons which increase their copy numbers in the genome through the homologous repair from sister strands [83, 84]. piRNAs repress TE activities by degrading mRNAs [56] or suppressing TE transcription through mediating heterochromatin formation $[135,141-143]$. Since it is still challenging to model the piRNA-mediated suppressive effect on target TE transcription quantitatively, here we only considered the repressive effects of piRNAs by degrading target mRNAs. A scheme of the TE:piRNA interaction in our model is presented in Fig. 5 a.

To expedite the simulations, the parameters optimized for $D$. melanogaster were scaled by 100 , as previously described [99] (see the legend of Fig. 5 for details). The different parameter settings and combinations were performed in 200 replicates. The simulations were initiated by assuming $10 \%$ of the individuals carrying the one TE randomly (Methods).

\section{The evolutionary arms race between TEs and piRNAs revealed by simulations}

To investigate the relative contributions of the factors in shaping the dynamics of TEs and piRNAs, we fixed the scaled parameters such as the replication rate $(u=0.03)$, the effective population size $\left(N_{e}=5000\right)$, the duplication rate $(d=0.003)$, the excision/inactivation rate $(i=0.001)$, the recombination rate $\left(r=10^{-8}\right.$ per nucleotide), the escape rate $(e=0)$, the penalty of excessive piRNAs $(p=0.5)$, the constants $a=10^{-3}$ and $b=5 \times 10^{-4}$. Although the size of the piRNA loci accounts for $\sim 5 \%$ of the euchromatin of D. melanogaster [56], many de novo piRNAs are generated outside the piRNA loci after a novel TE insertion [71, 94-96]. Therefore, we arbitrarily set $f$, the probability that a newly inserted TE is a piRNA-generation site, at 0.05 or 0.2 in our simulations. We varied the piRNA repression efficiency parameter $R(0,0.2,4,12$, and 20$)$ and the selection scaling factor $s(0.5,2,5,10$, and 15$)$ to explore the relationships between TEs and piRNAs in the populations.

Since the fitness cost of TEs has an exponential quadratic function $[139,140]$, TEs accumulate rapidly in the population and ultimately cause the extinction of the host organism if natural selection is weak $(s=0.5$, Additional file 1: Figure S12). By contrast, when the selection is very strong $(s=20)$, TEs are quickly removed from the population (Additional file 1: Figure S12). The outcomes of these two scenarios are very similar to the "one-side wins" scenario of inter-species evolutionary arms races, except that TEs are part of the host genomes. As expected under the traditional replication-selection model 


\section{a}
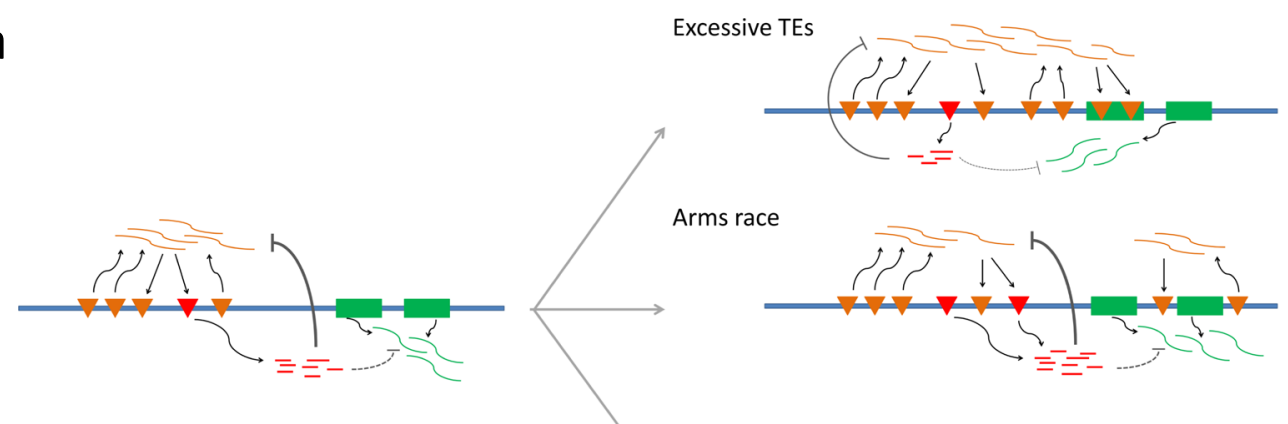

Excessive piRNAs
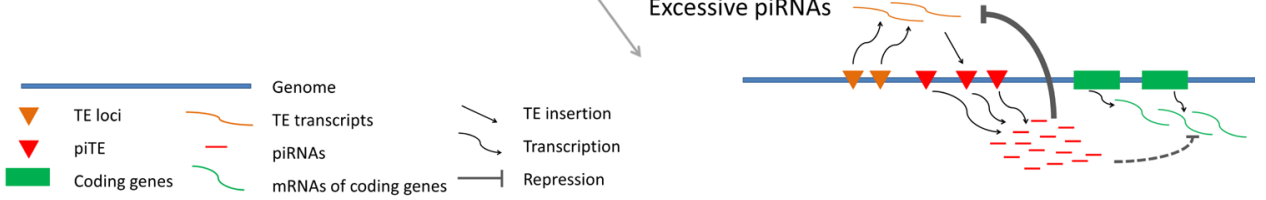

b
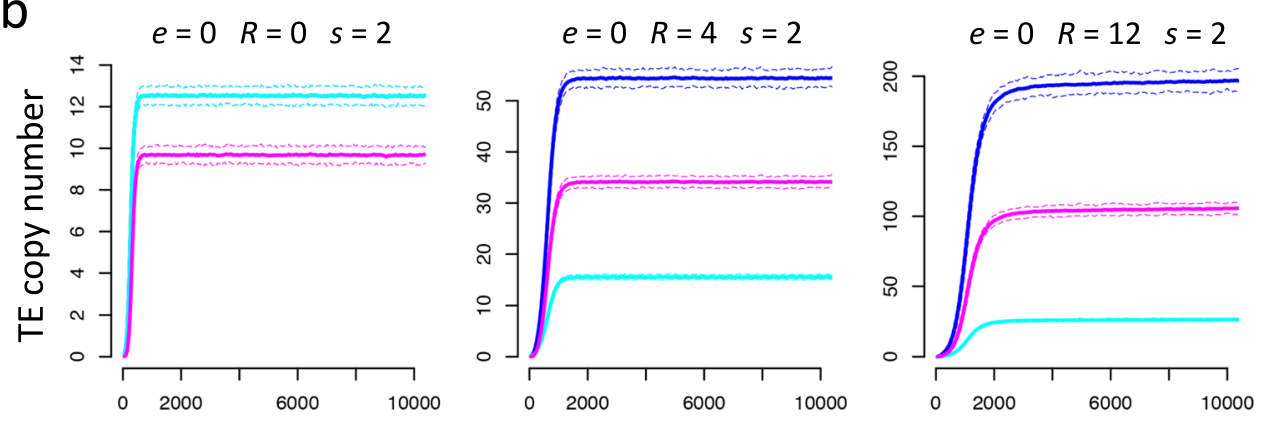

C
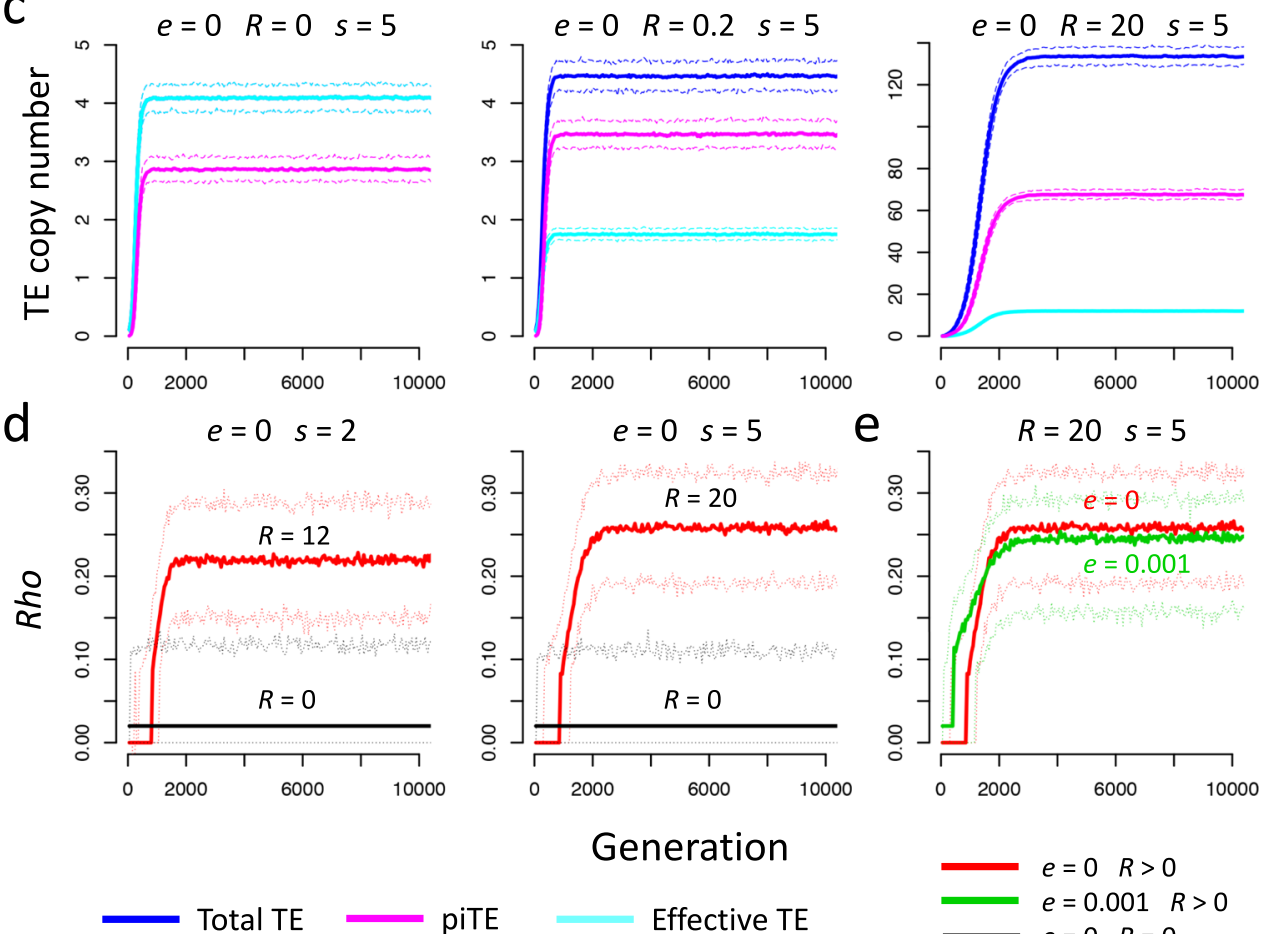

\section{Generation}

Effective TE

Fig. 5 (See legend on next page.) 


\section{(See figure on previous page.)}

Fig. 5 The evolutionary arms race between TEs and piRNAs revealed by simulations. a A schematic diagram illustrating the process and consequence of TE:piRNA interactions. Three possible consequences of TE:piRNA interactions depend on TE replication rate, the repressive strength of piRNAs on TEs, and the strength of purifying selection against TEs: 1) Excessive TEs. When TE replication rate is high and the repressive strength of piRNA is weak (TEs jumping into piRNA cluster and become piRT producing piRNAs), TEs soon become excessive in the genome, disrupt coding genes and have detrimental effects on the genome. 2) Arms race. When more piRTs produce more piRNAs and have stronger repression on TE, TE replication rate becomes lower and less TE exists in the genome, but the piRNA also alleviate detrimental effects of TEs on the genome. 3) Excessive piRNAs. If piRNA repression is very strong, TE activity becomes quite low and hardly jumps in the genome. Note that excessive dosage of piRNAs might cause off-target effects on the normal mRNAs and hence reduce the fitness of the host organism (dashed lines). The width of the lines represents the repression strength of piRNAs. $\mathbf{b}-\mathbf{c}$ The numbers ( $y$-axis) of TEs (blue), piTEs (pink), effective TEs (cyan) accumulated in one chromosome along with the generations ( $x$-axis) in the simulations. Under the same selection scaling factor ( $s=2$ for $\mathbf{b}$ and $s=5$ for $\mathbf{c}$ ), higher numbers of TEs, piTEs, and the effective TEs carried by one chromosome were observed when the repressiveness of piRNAs $(R)$ on TEs gets stronger. $\mathbf{d}$ Stronger repression of piRNA on the activities of TEs cause a positive correlation between piRNAs and TEs. The thick red lines are the mean Spearman's Rho ( $y$-axis) between the abundance of piRNAs and TEs along generations ( $x$-axis) in the simulations under $R=12$ (left) or $R=20$ (right). The thin dashed red lines are the 2.5 to $97.5 \%$ quantiles obtained in simulations. The black lines are Spearman's Rho under $R=0$. Since in both cases, the median (thick black) and the $2.5 \%$ (thin black) quantiles are both zero, and the $97.5 \%$ (thin black) quantile is displayed. e Escaping of TEs from piRNA repression ( $e=0.001$, green compared with $e=0$, red) decreases the positive correlation between the copy numbers of TEs and matched piRNAs. In all of these simulations, the following parameters are used: $u=0.03, N_{e}=5000, d=0.003, i=0.001$, $r=10^{-8}, p=0.5, a=10^{-3}, b=5 \times 10^{-4}, f=0.2, e=0$ in $\mathbf{b}-\mathbf{d}$. The $R$ and $s$ values are displayed on each panel. The correlation was calculated in 1000 sampled chromosomes that have at least one TE from the populations. All simulations were performed for 200 replicates

[20, 27-29], the numbers of TEs carried by one chromosome reaches equilibrium in the population when the intensity of natural selection is intermediate $(s=2$, Fig. $5 \mathrm{~b}$; $s=5$, Fig. 5c). Notably, the dynamics of piRNA copy number carried by one chromosome are similar to the dynamics of TEs located on the same chromosome (Fig. 5b, c). This is not surprising since in our simulations the biogenesis of piRNAs is dependent on the abundance of TEs.

To investigate whether piRNA-mediated repression of TE activities would generate a positive correlation between piRNAs and TEs, in the simulations we varied the $R$ parameter, which reflects the effectiveness of piRNA repression on the activities of TEs, while keeping the other parameters fixed. At $R=0$, when we sampled 1000 chromosomes that have at least one TE from the populations to calculate the correlation between TEs and piRNAs, we found only very weak positive correlation between the numbers of TEs and piRNAs located on the same chromosome (the median value Pearson's $r$ is 0 , Fig. $5 \mathrm{~d}$ ). These results suggest that although piRNAs depend on TE insertions in biogenesis, this alone would not produce a strong positive correlation between the numbers of piRNAs and TEs accumulated in each chromosome if piRNAs do not repress TEs effectively. However, when $R$ is increased, the correlation coefficient between TEs and piRNAs significantly increases after 1000 generations in the simulations ( $R=12, s=2 ; R=20, s=5$; Fig. $5 \mathrm{~d}$ ). These results indicate that stronger repression of TEs by piRNAs would yield a stronger positive correlation between TEs and piRNAs, since the deleterious effects of TEs would be alleviated by piRNA repression. Since mutations in TE sequences might cause a TE to escape the repression mediated by piRNAs, we also set $e=0.001$ to examine the extent to which TE escaping from piRNA repression would affect the correlation. Although we still observed a significant positive correlation between the copy numbers of TEs and matched piRNAs (green, Fig. 5e), the correlation coefficient is smaller than that obtained with $e=0$ (red, Fig. 5e). Therefore, mutations in TE target sites could potentially weaken the positive correlation between TEs and piRNAs. All the above results were obtained under the assumption that the probability that the insertion site of a novel TE is a piRNA-generating locus $(f)$ is 0.2 . To examine the extent to which the parameter $f$ affects the population dynamics of TEs and piRNAs, we also set $f=0.05$. If the repressiveness of piRNAs on TEs is strong $(R=20)$, we obtained very similar patterns when we set $f=0.2$ or $f=0.05$ (Additional file 1: Figure S13). In summary, our simulations suggest that three parameters could affect outcomes of the TE:piRNA interactions. First, the strength of natural selection is important: weak selective pressures would cause TEs to accumulate in the genomes and ultimately cause the extinction of the organisms, whereas strong natural selection would result in elimination of TEs from the population. Second, the repressiveness of piRNAs on TEs affects the arms race patterns. Third, the escaping rate of TEs from piRNA-mediated suppression would decrease the positive correlation between TEs and piRNAs.

In summary, our results suggest that if TEs can persist in the population in the long-run, the interactions between TEs and piRNAs could lead to an evolutionary arms race.

\section{Conclusions}

piRNAs repress target TE activities by degrading mRNAs or inhibiting TE transcription [135, 141-143]. Besides piRNAs, many epigenetic factors affecting the transcription of the piRNA clusters, such as the epigenetic modifications of chromatin states $[96,144]$ and the interactions 
between the Rhino complex with the H3K9me3-marked chromatin [70, 71]. Moreover, the piRNA-mediated spread of heterochromatin from TEs into neighboring genes might disrupt the function of those genes and cause deleterious effects [115]. In this study, we only considered the repressive effects of piRNAs by degrading target mRNAs because quantitative modeling piRNA-mediated suppression of TE transcription is still challenging at this moment. However, since the piRNA-mediated transcriptional suppression of target TEs are also based on the sequence matching between piRNAs and target TEs, we expect that the evolutionary arms race signals also exist in the piRNA:TE interactions through this mechanism. More complete understanding of the TE and piRNA biology is needed to provide a thorough picture of TE:piRNA interactions in the future studies.

Many organisms have developed diverse mechanisms to repress TEs. The molecular mechanisms underlying an evolutionary arms race are important for understanding the origin and evolution of genetic and phenotypic diversities. Due to the uniqueness of piRNA biogenesis and their clearly repressive effects on TE transposition, the TE: piRNA interaction system gives us a new opportunity to detect a potentially widespread evolutionary arms race in nature. Although the TE:piRNA interaction shares similarities with the CRISPR/Cas9 system [145] in that the emergence of the suppressor elements is dependent on the invasive elements, the difference is that in the former piRNAs repress TEs by degrading mRNAs or inhibiting transcription whereas in the latter the invasive DNA fragments are destroyed. Thus, the interactions between piRNAs and TEs provide novel insights into the biology of the arms race between genomic parasites and hosts.

Understanding the population dynamics of TEs and the underlying evolutionary forces has been a research objective pursued by many evolutionary biologists [146]. Although the piRNA pathways are crucial in suppressing the activities of TEs [56], whether there is an evolutionary arms race between TEs and piRNAs was unclear [31]. In this study, we detected significantly positive Spearman's correlations between TEs and antisense piRNAs for six TE families. Our simulations further highlight that TE activities and the strength of purifying selection against TEs are important factors shaping the interactions between TEs and piRNAs. It is possible that the piRNA repression would alleviate the deleterious effects of TEs, which causes TEs to keep increasing in the genomes. Our studies also suggest that de novo generation of piRNAs is an important mechanism to repress the newly invaded TEs. Although the interactions between TEs and piRNAs are complex and many factors should be considered to impact their interaction dynamics, our results suggest the emergence, repression specificity and strength of piRNAs on TEs should be considered in studying the landscapes of TE insertions in Drosophila.

\section{Methods}

\section{Drosophila stocks and fly husbandry}

The Global Diversity Lines (GDL) strains of D. melanogaster with whole-genome sequences were collected from five continents [110]. Genome information of 81 of these strains sequenced with Illumina 100 bp paired-end protocols was analyzed in this study. These strains were sampled from: Beijing, China (14 lines, abbreviated B); Ithaca, NY USA (17 lines, abbreviated I); Netherlands, Europe (19 lines, abbreviated N); Tasmania, Australia (17 lines, abbreviated T); and Zimbabwe, Africa (14 lines, abbreviated Z). All flies were maintained on standard yeast-cornmealdextrose medium at $25^{\circ} \mathrm{C}$. We chose two strains with the highest genome coverage from each population (B10, B12, I06, I17, N10, N16, T05, T07, ZW155, and ZW184) for mRNA and small RNA sequencing.

\section{RNA preparation and library construction}

The ovaries of 3-5 day old female flies were dissected in Ringer's solution and kept in RNAlater (Ambion) before RNA extraction. Total RNA was extracted with TRIzol reagent (Invitrogen) according to the manufacturer's instructions. Total RNA was treated with DNaseI (Takara) before mRNA-seq library construction. The purity and concentration of RNA were validated with NanoDrop and Fragment Analyzer (AATI). The cloning of small RNAs was conducted following the procedures described previously [137]. The small RNAs of 18-30 nt were gel purified. Next, the small RNAs were subjected to ligation, reverse transcription and PCR. Sequencing was done with Illumina HiSeq-2500 sequencer (run type: single-end; read length: $50 \mathrm{nt}$ ).

\section{TE content and insertion analysis}

The DNA NGS reads were filtered by trimmomatic [147]. DNA sequences were all mapped to the reference genome of D. melanogaster (FlyBase Release 6 or 5.57, www.FlyBase.org) with bwa [148], and mapped to TE sequences annotated in BDGP TE dataset (www.fruitfly.org) and RepBase Update (www.girinst.org/repbase) [123] with BLAT [124].

We employed two complementary approaches to identify and quantify TE polymorphism. First, for the TE insertions annotated in the reference genome of $D$. melanogaster, we only considered the 3544 TE insertions that have boundary sequences uniquely mapped to the reference genome. For the paired-end reads in each strain, we required 1) the paired-end reads to be properly mapped to the reference genome, 2) one read spanning at least $30 \mathrm{bp}$ flanking one boundary site of one TE insertion, 3) the mapped sequences having no more than 4 (out of 100) mismatches (or indels) with the reference genomes, 4) the TE insertion was not detected as "Absence" in the TEMP package [111]. We employed TEMP 
[111] to systematically screen possible novel TE insertions in the GDL strains that were absent in the reference genome. The TE references were all the possible TE sequences from the BDGP TE dataset, Repbase Update, and FlyBase. Only the insertions by the putative functional TE and TE clusters which were filtered by 95\% identity with usearch [149] were retained. The insertions located less than $100 \mathrm{bp}$ away were merged. We further required the following criteria to be met in at least one strain: 1) The new insertions should have supporting evidence in both flanking sides, and 2) The frequency of insertions should exceed $80 \%$ of the total number of reads spanning the TE insertion sites. The clustering of TE copy number and TE insertions was done with Multiple Dimensional Scaling [150].

\section{Population parameter calculation}

The SNPs of the GDL strains were obtained from Grenier et al. [110]. The population parameters $\theta_{\pi}$, Tajima's $D$ [116], and Fay and Wu $H$ [117] were calculated from the called SNPs. SNPs were filtered if the missing value $>50 \%$ and only bi-allele SNPs were chosen. $\theta_{\pi}$ and Tajima's $D$ were calculated with vcftools [151]. SNP annotations were done with snpEff [152]. The genomes of $D$. simulans, D. sechellia and D. yakuba were used to find the ancestral SNP allele. The SNPs in D. melanogaster were converted by liftover [153]. Fay and Wu' $H$ test was calculated by Fay's $C$ code [117]. The composite likelihood ratio (CLR) [118-120] was calculated with a grid size of 1 (or 10) kb with SweeD [121]. Since the accurate demographic history of each local population and the global population remains unknown, we used the default parameter settings in SweeD. In each local or the global population analysis, the CLR values of SweeD were ranked for each chromosome. LD plots were plotted with Haploview [154].

\section{RNA expression analysis}

mRNA sequences were aligned to the genome (FlyBase r5.57) with TopHat2 [155] with 2 mismatches. Gene read counts were done with HTseq-count [156]. mRNA reads were mapped to the canonical TE sequences with STAR [157]. The fold change in gene expression level induced by TE insertion is calculated from the ratio between the gene expression in the strains with TE insertion and in the strains without TE insertions.

\section{Small RNA analysis}

We deep-sequenced small RNAs from ovaries of 10 Global Diversity Lines (GDL) strains of D. melanogaster and collected the ovarian small RNA-Seq data of 16 DGRP (Drosophila Genetic Reference Panel) strains from Song et al. [95]. For these small RNA-Seq data, the 3'-adaptor sequences were removed using the Cutadapt software
[158]. The trimmed small RNA reads that are shorter than 18 nts were discarded. The small RNAs were mapped to the reference genome of D. melanogaster (FlyBase r5.57), the TE sequences in the BDGP TE dataset and RepBase using Bowtie2 [159]. In case a small RNA read was mapped on multiple locations, it was equally split across these locations. After removing reads mapped on rRNAs, tRNAs, miscRNAs, ncRNAs and miRNAs that were annotated in FlyBase (r5.57), the remaining small RNAs ranged from 23 to 29 nts are treated as putative piRNAs. For each strain, we normalized the 20-22 nt siRNAs that were mapped to TEs and the 23-29 nt piRNAs that were mapped on the reference genome and TEs to one million. The RPKM of piRNAs on each TE was calculated as (total weighted piRNAs on that TE)/(length of that TE) $\times 10^{9} /($ total $23-29$ nt small RNA reads and 20-22 nt reads mapped to TEs). The ping-pong signals were identified with the Python script that was previously described [160].

We predicted the target of piRNAs by requiring perfect antisense matching between position 2-11 of a 23-29 nt piRNA and a TE sequence. In case a piRNA has multiple target sites, we equally split the piRNA to all the target sites. Then for each TE sequence, we calculated the weighted abundance of piRNAs that target that TE.

The de novo piRNA production signature in the flanking regions of the novel TE insertion was defined similarly as a previous study [95] and with the following requirements. (1) In the flanking 2-kb regions of the novel TE insertion, the abundance of piRNA $\geq 0.5$ RPKM; (2) the antisense piRNAs in the upstream flanking region and the sense piRNAs in the downstream flanking region consisted of at least $70 \%$ of the total piRNAs.

\section{DNA copy number of TEs}

We collected the Illumina paired-end DNA-Seq reads of 10 GDL and 16 DGRP strains. We mapped DNA-Seq reads to the reference genome (FlyBase r5.57) and TE sequences (a combination of FlyBase, BDGP, and RepBase) with bwa [148], respectively. We discarded the reads with only one mate mapped to the reference sequence (less than $2 \%$ on average). For each TE sequence, we calculated the coverage of DNA-Seq on each position with bedtools [161]. The median coverage values of the reads-covered sites were assigned to each TE. To exclude the potential bias caused by the different read length and sequencing depth, we also calculated the median coverage for all the autosomal single-copy genes. In each library, the median coverage for each TE was normalized by the median coverage of single-copy genes. The ratios obtained were regarded as the copy number of TEs. Note that the active part of the P-element (positions 819-2527, GenBank Accession number X06779) was extracted as an individual sequence and analyzed separately. 


\section{Simulation}

The forward simulations were performed following a similar approach as we previously described [99]. Briefly, the simulation begins with $N_{e}$ (5000) diploid individuals, in which $10 \%$ of the individuals have a single TE insertion of the sample type. In each generation, two individuals were randomly selected (based on their fitness) as the parents of an offspring individual. Recombination $(r)$, changing sequences to evolve into a new subtype (escaping, $e$ ), excision $(i)$, and duplication $(d)$ of TEs and piRNAs occur during meiosis. In a parent individual, a TE retrotransposes to new positions in the genome at a rate $u /\left(1+R \cdot \frac{y_{j}}{x_{j}}\right)$, where $R$ is a constant, $x_{j}$ and $y_{j}$ is the number of TEs and piRNAs of the same type in that individual, respectively. For each new TE insertion, it has $f$ change to become a piRNA-generating locus. Only the TE that does not generate piRNAs can retrotranspose. The simulation was performed for 15,000 generations. For each parameter (or parameter combination), the whole simulation process was replicated 200 times. A simulation stops when all TE copies are purged from the population or the average fitness of the individuals is smaller than 0.05 . The correlation coefficients between the copy number of TE and piRNAs of all subtypes carried in one chromosome was calculated in 1000 sampled chromosomes that have at least one TE from the populations. The correlation coefficient is not calculated when the number of individuals that have at least one $\mathrm{TE}$ is smaller than 1000. In case the correlation is not statistically significant in a test $(P>0.05)$, the correlation coefficient is set at 0 .

\section{Supplementary information}

Supplementary information accompanies this paper at https://doi.org/10 1186/s12862-020-1580-3.

\section{Additional file 1: Figure S1. Tajima's D in $10 \mathrm{~kb}$ bins in each} population. Figure S2. Fay and Wu's H in $10 \mathrm{~kb}$ bins in each population. Figure S3. The composite likelihood ratio (CLR) with a grid size of $10 \mathrm{~kb}$ in each population. Figure S4. Signatures of natural selection on the novel TE insertion located on chr3R: 15380492-15,380,496 in N population. Figure S5. Multidimensional scaling (MDS) of known (a) or novel (b) TE insertions across GDL strains. Figure S6. Percentages of reads (y-axis) that are mapped to all the sources of TE sequences with different map length and identity. Figure S7. Coverage of weighted piRNAs mapped to the sense (red) and anti-sense (blue) of piRNA cluster flamenco in $10 \mathrm{GDL}$ strains. Figure S8. Coverage of weighted piRNAs mapped to the sense (red) and anti-sense (blue) of piRNA cluster 42AB in $10 \mathrm{GDL}$ strains. Figure S9. Barplots showing the RPKMs of de novo piRNAs generated in the flanking region $(2 \mathrm{~kb}$ ) of novel TE insertions across $10 \mathrm{GDL}$ strains. Figure S10. Barplots showing the RPKMs of de novo siRNAs generated in the flanking region (2 kb) of novel TE insertions. Figure S11. Longer TEs tend to be targeted by higher densities of piRNAs in the 10 GDL strains. Figure S12. The fitness of host organisms (left) and number of TEs carried by one chromosome when selection is weak ( $s=0.5$, upper) or strong ( $s=20$, lower). Figure $\mathbf{S 1 3}$. Numbers ( $y$-axis) of TEs (blue), piTEs (pink, these are TEs that are piRNA-repressed), effective TEs (cyan) accumulated in one chromosome along the generations (x-axis) in the simulations. Table S1. Genome features of all novel TE insertions on all chromosomes. Table S2. Candidate hitchhiking events associated with TE insertions in local populations. Table S3. Mapping summary of sequenced small RNAs in the $10 \mathrm{GDL}$ and 16 DGRP strains.

Additional file 2: Table S4. Statistics of correlation analysis between piRNA abundance and TE copy number in the $10 \mathrm{GDL}$ and 16 DGRP strains.

\section{Acknowledgments}

We thank Biodynamic Optical Imaging Center from Peking University for the sequencing services. We also thank Mr. Jie Zhang for technical support in data analysis. Part of the analysis was performed on the High Performance Computing Platform of the Center for Life Science.

\section{Authors' contributions}

Conceptualizations: JL and AGC; Methodology: SL, HZ, and YD; Formal analysis: SL, XY, and YD; Investigation: $S L$ and $Y D$; Writing: $J$ and $A G C$; Supervision: $J$ and AGC; Funding acquisition: $J$ and AGC. All authors read and approved the final manuscript.

\section{Funding}

This work was supported by grants from National Natural Science Foundation of China (Nos. 91731301, 91431101, 31571333, and 31771411) to Jian Lu, and NIH R01 grant (GM119125) to AGC and D. Barbash. The funding bodies played no role in the design of the study and collection, analysis, and interpretation of data and in writing the manuscript.

\section{Availability of data and materials}

The datasets generated and analysed during the current study are available in the NCBI Sequence Read Archive (SRA) under accession number

SRP068882, and from Song et al. [95] in NCBI SRA under accession number SRP019948.

Ethics approval and consent to participate

Not applicable.

\section{Consent for publication}

Not applicable.

\section{Competing interests}

The authors declare that they have no competing interests.

\section{Author details}

${ }^{1}$ State Key Laboratory of Protein and Plant Gene Research, Center for Bioinformatics, College of Life Sciences and Peking-Tsinghua Center for Life Sciences, Peking University, Beijing 100871, China. ${ }^{2}$ College of Plant Protection, Beijing Advanced Innovation Center for Food Nutrition and Human Health, China Agricultural University, Beijing 100193, China.

${ }^{3}$ Academy for Advanced Interdisciplinary Studies, Peking University, Beijing 100871, China. ${ }^{4}$ Department of Molecular Biology and Genetics, Cornell University, Ithaca, NY 14853, USA.

Received: 21 July 2019 Accepted: 13 January 2020

Published online: 28 January 2020

References

1. Van Valen L. A new evolutionary law. Evol Theory. 1973;1:1-30.

2. Neiman M, Fields P. Antagonistic interspecific coevolution. In: Kliman R, editor. Encyclopedia of Evolutionary Biology, vol. 1. Oxford: Academic Press; 2016. p. 93-100.

3. Dawkins R, Krebs JR. Arms races between and within species. Proc R Soc Lond B Biol Sci. 1979:205(1161):489.

4. Hurst LD, Atlan A, Bengtsson BO. Genetic conflicts. Q Rev Biol. 1996;71(3): 317-64

5. Werren JH. Selfish genetic elements, genetic conflict, and evolutionary innovation. Proc Natl Acad Sci U S A. 2011;108:10863-70.

6. Rice WR. Nothing in genetics makes sense except in light of genomic conflict. Annu Rev Ecol Evol Syst. 2013:44(1):217-37.

7. Crespi B, Nosil P. Conflictual speciation: species formation via genomic conflict. Trends Ecol Evol. 2013;28(1):48-57. 
8. Lee YCG, Langley CH. Transposable elements in natural populations of Drosophila melanogaster. Philos T R Soc B. 2010;365(1544):1219.

9. Luo S, Lu J. Silencing of transposable elements by piRNAs in Drosophila: an evolutionary perspective. Genomics Proteomics Bioinformatics. 2017;15(3): 164-76

10. Britten RJ, Kohne DE. Repeated sequences in DNA. Science. 1968;161(3841): 529.

11. Calos MP, Miller JH. Transposable elements. Cell. 1980;20(3):579-95.

12. Finnegan DJ. Eukaryotic transposable elements and genome evolution. Trends Genet. 1989;5(4):103-7.

13. Kidwell MG, Lisch DR. Transposable elements and host genome evolution. Trends Ecol Evol. 2000;15(3):95-9.

14. Levin HL, Moran JV. Dynamic interactions between transposable elements and their hosts. Nat Rev Genet. 2011;12(9):615-27.

15. Slotkin RK, Martienssen R. Transposable elements and the epigenetic regulation of the genome. Nat Rev Genet. 2007;8(4):272-85.

16. Wicker T, Sabot F, Hua-Van A, Bennetzen JL, Capy P, Chalhoub B, Flavell A, Leroy $\mathrm{P}$, Morgante $\mathrm{M}$, Panaud $\mathrm{O}$, et al. A unified classification system for eukaryotic transposable elements. Nat Rev Genet. 2007:8(12):973-82.

17. Rebollo R, Romanish MT, Mager DL. Transposable elements: an abundant and natural source of regulatory sequences for host genes. Annu Rev Genet. 2012;46(1):21-42.

18. Cuomo CA, Guldener U, Xu JR, Trail F, Turgeon BG, Di Pietro A, Walton JD, Ma LJ, Baker SE, Rep M, et al. The Fusarium graminearum genome reveals a link between localized polymorphism and pathogen specialization. Science. 2007;317(5843):1400-2.

19. Schnable PS, Ware D, Fulton RS, Stein JC, Wei F, Pasternak S, Liang C, Zhang J, Fulton L, Graves TA, et al. The B73 maize genome: complexity, diversity, and dynamics. Science. 2009;326(5956):1112-5.

20. Charlesworth B, Charlesworth D. The population dynamics of transposable elements. Genet Res. 1983;42(01):1-27.

21. Finnegan DJ. Transposable elements. Curr Opin Genet Dev. 1992;2(6):861-7.

22. McDonald JF, Matyunina LV, Wilson S, Jordan IK, Bowen NJ, Miller WJ. LTR retrotransposons and the evolution of eukaryotic enhancers. Genetica. 1997; 100(1-3):3-13.

23. Puig M, Cáceres M, Ruiz A. Silencing of a gene adjacent to the breakpoint of a widespread Drosophila inversion by a transposon-induced antisense RNA. Proc Natl Acad Sci U S A. 2004;101(24):9013-8.

24. Sentmanat MF, Elgin SCR. Ectopic assembly of heterochromatin in Drosophila melanogaster triggered by transposable elements. Proc Natl Acad Sci U S A. 2012;109(35):14104-9.

25. Brookfield JF. Models of repression of transposition in PM hybrid dysgenesis by $P$ cytotype and by zygotically encoded repressor proteins. Genetics. 1991;128(2):471-86.

26. Nuzhdin SV. Sure facts, speculations, and open questions about the evolution of transposable element copy number. Genetica. 1999;107(1-3):129-37.

27. Montgomery EA, Langley $\mathrm{CH}$. Transposable elements in mendelian populations. II. Distribution of three COPIA-like elements in a natural population of Drosophila melanogaster. Genetics. 1983;104(3):473-83.

28. Langley $\mathrm{CH}$, Montgomery E, Hudson R, Kaplan N, Charlesworth B. On the role of unequal exchange in the containment of transposable element copy number. Genet Res. 1988;52(03):223-35.

29. Charlesworth $\mathrm{B}$, Langley $\mathrm{CH}$. The population genetics of Drosophila transposable elements. Annu Rev Genet. 1989;23:251-87.

30. Petrov DA, Aminetzach YT, Davis JC, Bensasson D, Hirsh AE. Size matters: non-LTR retrotransposable elements and ectopic recombination in Drosophila. Mol Biol Evol. 2003;20(6):880-92.

31. Petrov DA, Fiston-Lavier A-S, Lipatov M, Lenkov K, González J. Population genomics of transposable elements in Drosophila melanogaster. Mol Biol Evol. 2011;28(5):1633-44.

32. Karess RE, Rubin GM. Analysis of P transposable element functions in Drosophila. Cell. 1984;38(1):135-46.

33. Ohare K, Rubin GM. Structures of $P$ transposable elements and their sites of insertion and excision in the Drosophila melanogaster genome. Cell. 1983; 34(1):25-35

34. Pimpinelli S, Berloco M, Fanti L, Dimitri P, Bonaccorsi S, Marchetti E, Caizzi R, Caggese C, Gatti M. Transposable elements are stable structural components of Drosophila melanogaster heterochromatin. Proc Natl Acad Sci U S A. 1995;92(9):3804-8.

35. Rubin GM, Spradling AC. Genetic-transformation of Drosophila with transposable element vectors. Science. 1982;218(4570):348-53.
36. Quesneville H, Bergman CM, Andrieu O, Autard D, Nouaud D, Ashburner M, Anxolabehere D. Combined evidence annotation of transposable elements in genome sequences. PLoS Comp Biol. 2005;1(2):166-75.

37. Drosophila 12, Genomes C, Clark AG, Eisen MB, Smith DR, Bergman CM, Oliver B, Markow TA, Kaufman TC, Kellis M, Gelbart W, et al. Evolution of genes and genomes on the Drosophila phylogeny. Nature. 2007;450(7167): 203-18.

38. Bergman CM, Quesneville $H$, Anxolabéhère $D$, Ashburner M. Recurrent insertion and duplication generate networks of transposable element sequences in the Drosophila melanogaster genome. Genome Biol. 2006; 7(11):R112.

39. Kapitonov W, Jurka J. Molecular paleontology of transposable elements in the Drosophila melanogaster genome. Proc Natl Acad Sci U S A. 2003; 100(11):6569-74.

40. Kaminker JS, Bergman CM, Kronmiller B, Carlson J, Svirskas R, Patel S, Frise E, Wheeler DA, Lewis SE, Rubin GM, et al. The transposable elements of the Drosophila melanogaster euchromatin: a genomics perspective. Genome Biol. 2002;3(12):research0084 0081.

41. Smith CD, Shu S, Mungall CJ, Karpen GH. The release 5.1 annotation of Drosophila melanogaster heterochromatin. Science. 2007;316(5831):1586.

42. Ashburner M. Drosophila. A laboratory handbook. Cold Spring Harbor: Cold Spring Harbor Laboratory Press; 1989.

43. Gifford WD, Pfaff SL, Macfarlan TS. Transposable elements as genetic regulatory substrates in early development. Trends Cell Biol. 2013;23(5):21826.

44. Li W, Prazak L, Chatterjee N, Grüninger S, Krug L, Theodorou D, Dubnau J. Activation of transposable elements during aging and neuronal decline in Drosophila. Nat Neurosci. 2013;16(5):529-31.

45. Mateo L, Ullastres A, González J. A transposable element insertion confers xenobiotic resistance in Drosophila. PLoS Genet. 2014;10(8):e1004560.

46. Kofler R, Betancourt AJ, Schlötterer C. Sequencing of pooled DNA samples (Pool-Seq) uncovers complex dynamics of transposable element insertions in Drosophila melanogaster. PLoS Genet. 2012;8(1):e1002487.

47. González J, Lenkov K, Lipatov M, Macpherson JM, Petrov DA. High rate of recent transposable element-induced adaptation in Drosophila melanogaster. PLoS Biol. 2008;6(10):e251.

48. Chen B, Shilova WY, Zatsepina OG. Evgen\&apos;ev MB, Feder ME: location of $\mathrm{P}$ element insertions in the proximal promoter region of Hsp70A is consequential for gene expression and correlated with fecundity in Drosophila melanogaster. Cell Stress Chaperones. 2008;13(1):11-7.

49. Aminetzach YT, Macpherson JM, Petrov DA. Pesticide resistance via transposition-mediated adaptive gene truncation in Drosophila. Science. 2005;309(5735):764-7.

50. Cridland JM, Thornton KR, Long AD. Gene expression variation in Drosophila melanogaster due to rare transposable element insertion alleles of large effect. Genetics. 2015;199(1):85.

51. Girard A, Sachidanandam R, Hannon GJ, Carmell MA. A germline-specific class of small RNAs binds mammalian Piwi proteins. Nature. 2006;442(7099): 199-202.

52. Grivna ST, Beyret E, Wang Z, Lin HF. A novel class of small RNAs in mouse spermatogenic cells. Genes Dev. 2006;20(13):1709-14.

53. Lau NC, Seto AG, Kim J, Kuramochi-Miyagawa S, Nakano T, Bartel DP, Kingston RE. Characterization of the piRNA complex from rat testes. Science. 2006;313(5785):363-7.

54. Saito K, Nishida KM, Mori T, Kawamura Y, Miyoshi K, Nagami T, Siomi H, Siomi MC. Specific association of Piwi with rasiRNAs derived from retrotransposon and heterochromatic regions in the Drosophila genome. Genes Dev. 2006;20(16):2214-22.

55. Vagin W, Sigova A, Li CJ, Seitz H, Gvozdev V, Zamore PD. A distinct small RNA pathway silences selfish genetic elements in the germline. Science. 2006:313(5785):320-4.

56. Brennecke J, Aravin AA, Stark A, Dus M, Kellis M, Sachidanandam R, Hannon GJ. Discrete small RNA-generating loci as master regulators of transposon activity in Drosophila. Cell. 2007;128(6):1089-103.

57. Ruby JG, Jan C, Player C, Axtell MJ, Lee W, Nusbaum C, Ge H, Bartel DP. Large-scale sequencing reveals 21U-RNAs and additional microRNAs and endogenous siRNAs in C. elegans. Cell. 2006;127(6):1193-207.

58. Houwing S, Kamminga LM, Berezikov E, Cronembold D, Girard A, van den Elst H, Filippov DV, Blaser H, Raz E, Moens CB, et al. A role for Piwi and piRNAs in germ cell maintenance and transposon silencing in Zebrafish. Cell. 2007;129(1):69-82. 
59. Aravin AA, Naumova NM, Tulin AV, Vagin W, Rozovsky YM, Gvozdev VA. Doublestranded RNA-mediated silencing of genomic tandem repeats and transposable elements in the D. melanogaster germline. Curr Biol. 2001;11(13):1017-27.

60. Iwasaki YW, Siomi MC, Siomi H. PIWl-interacting RNA: its biogenesis and functions. Annu Rev Biochem. 2015;84:405-433.

61. Brennecke J, Malone CD, Aravin AA, Sachidanandam R, Stark A, Hannon GJ. An epigenetic role for maternally inherited piRNAs in transposon silencing. Science. 2008;322(5906):1387-92.

62. Juliano C, Wang JQ, Lin HF. Uniting germline and stem cells: the function of Piwi proteins and the piRNA pathway in diverse organisms. Annu Rev Genet. 2011;45:447-69.

63. Klattenhoff $C$, Theurkauf W. Biogenesis and germline functions of piRNAs. Development. 2008;135(1):3-9.

64. Senti KA, Brennecke J. The piRNA pathway: a fly's perspective on the guardian of the genome. Trends Genet. 2010;26(12):499-509.

65. Siomi MC, Sato K, Pezic D, Aravin AA. PIWl-interacting small RNAs: the vanguard of genome defence. Nat Rev Mol Cell Biol. 2011;12(4):246-58.

66. Thomson T, Lin HF. The biogenesis and function of PIWI proteins and piRNAs: progress and prospect. Annu Rev Cell Dev Biol. 2009:25:355-76.

67. Ozata DM, Gainetdinov I, Zoch A, O'Carroll D, Zamore PD. PIWl-interacting RNAs: small RNAs with big functions. Nat Rev Genet. 2019;20(2):89-108.

68. Mohn F, Handler D, Brennecke J. piRNA-guided slicing specifies transcripts for Zucchini-dependent, phased piRNA biogenesis. Science. 2015;348(6236):812-7.

69. Han BW, Wang W, Li C, Weng Z, Zamore PD. piRNA-guided transposon cleavage initiates Zucchini-dependent, phased piRNA production. Science. 2015;348(6236):817-21

70. Zhang Z, Wang J, Schultz N, Zhang F, Parhad SS, Tu S, Vreven T, Zamore PD, Weng Z, Theurkauf WE. The HP1 homolog rhino anchors a nuclear complex that suppresses piRNA precursor splicing. Cell. 2014;157(6):1353-63.

71. Mohn F, Sienski G, Handler D, Brennecke J. The rhino-deadlock-cutoff complex licenses noncanonical transcription of dual-strand piRNA clusters in Drosophila. Cell. 2014;157(6):1364-79.

72. Huang $X$, Fejes Toth $K$, Aravin AA. piRNA biogenesis in Drosophila melanogaster. Trends Genet. 2017:33(11):882-94.

73. Czech B, Munafo M, Ciabrelli F, Eastwood EL, Fabry MH, Kneuss E, Hannon GJ. piRNA-guided genome defense: from biogenesis to silencing. Annu Rev Genet. 2018:52:131-57.

74. Hayashi R, Schnabl J, Handler D, Mohn F, Ameres SL, Brennecke J. Genetic and mechanistic diversity of piRNA 3'-end formation. Nature. 2016; 539(7630):588-92.

75. Andersen PR, Tirian L, Vunjak M, Brennecke J. A heterochromatin-dependent transcription machinery drives piRNA expression. Nature. 2017;549(7670):54-9.

76. Hur JK, Luo Y, Moon S, Ninova M, Marinov GK, Chung YD, Aravin AA. Splicing-independent loading of TREX on nascent RNA is required for efficient expression of dual-strand piRNA clusters in Drosophila. Genes Dev. 2016;30(7):840-55.

77. Gunawardane LS, Saito K, Nishida KM, Miyoshi K, Kawamura Y, Nagami $\mathrm{T}$, Siomi H, Siomi MC. A slicer-mediated mechanism for repeatassociated siRNA 5 ' end formation in Drosophila. Science. 2007 315(5818):1587-90.

78. Czech B, Hannon GJ. One loop to rule them all: the ping-pong cycle and piRNA-guided silencing. Trends Biochem Sci. 2016;41(4):324-37.

79. Khurana JS, Wang J, Xu J, Koppetsch BS, Thomson TC, Nowosielska A, Li CJ, Zamore PD, Weng ZP, Theurkauf WE. Adaptation to P element transposon invasion in Drosophila melanogaster. Cell. 2011;147(7):1551-63.

80. Kidwell MG, Kidwell JF, Sved JA. Hybrid dysgenesis in Drosophila melanogaster - syndrome of aberrant traits including mutation, sterility and male recombination. Genetics. 1977;86(4):813-33.

81. Kidwell MG. Evolution of hybrid dysgenesis determinants in Drosophila melanogaster. Proc Natl Acad Sci U S A. 1983;80(6):1655-9.

82. Kelleher ES. Reexamining the P-element invasion of Drosophila melanogaster through the lens of piRNA silencing. Genetics. 2016;203(4):1513.

83. Engels WR, Johnson-Schlitz DM, Eggleston WB, Sved J. High-frequency P element loss in Drosophila is homolog dependent. Cell. 1990;62(3): 515-25.

84. Spradling AC, Bellen HJ, Hoskins RA. Drosophila P elements preferentially transpose to replication origins. Proc Natl Acad Sci U S A. 2011;108(38): 15948-53.

85. Aravin AA, Lagos-Quintana M, Yalcin A, Zavolan M, Marks D, Snyder B, Gaasterland T, Meyer J, Tuschl T. The small RNA profile during Drosophila melanogaster development. Dev Cell. 2003;5(2):337-50.
86. Malone CD, Brennecke J, Dus M, Stark A, McCombie WR, Sachidanandam R, Hannon GJ. Specialized piRNA pathways act in Germline and somatic tissues of the Drosophila ovary. Cell. 2009;137(3):522-35.

87. Nishida KM, Saito K, Mori T, Kawamura Y, Nagami-Okada T, Inagaki S, Siomi $\mathrm{H}$, Siomi MC. Gene silencing mechanisms mediated by Aubergine-piRNA complexes in Drosophila male gonad. RNA. 2007;13(11):1911-22.

88. Zanni V, Eymery A, Coiffet M, Zytnicki M, Luyten I, Quesneville H, Vaury C, Jensen S. Distribution, evolution, and diversity of retrotransposons at the flamenco locus reflect the regulatory properties of piRNA clusters. Proc Natl Acad Sci U S A. 2013;110(49):19842-7.

89. Chambeyron S, Popkova A, Payen-Groschêne G, Brun C, Laouini D, Pelisson A, Bucheton A. piRNA-mediated nuclear accumulation of retrotransposon transcripts in the Drosophila female germline. Proc Natl Acad Sci U S A. 2008;105(39):14964-9.

90. Orsi GA, Joyce EF, Couble P, McKim KS, Loppin B. Drosophila IR hybrid dysgenesis is associated with catastrophic meiosis and abnormal zygote formation. J Cell Sci. 2010;123(20):3515-24.

91. Kidwell MG. Hybrid dysgenesis in Drosophila melanogaster: nature and inheritance of P-element regulation. Genetics. 1985;111(2):337-50.

92. Bucheton A. Study of non Mendelian female sterility in Drosophila melanogaster. Hereditary transmission of efficiency degrees of the reactor factor. C R Acad Sci Hebd Seances Acad Sci D. 1973;276(4):641-4.

93. Bucheton A, Picard G. A partly inheritable aging influence on nonMendelian female sterility in Drosophila melanogaster. C R Acad Sci Hebd Seances Acad Sci D. 1975;281(14):1035-8.

94. Shpiz S, Ryazansky S, Olovnikov I, Abramov Y, Kalmykova A. Euchromatic transposon insertions trigger production of novel pi- and endo-siRNAs at the target sites in the Drosophila germline. PLoS Genet. 2014;10(2): e1004138.

95. Song J, Liu J, Schankenberg S, Ha H, Xing J, Chen KC. Variation in piRNA and transposable element content in strains of Drosophila melanogaster. Genome Biol Evol. 2014;6:2786-2798.

96. Olovnikov I, Ryazansky S, Shpiz S, Lavrov S, Abramov Y, Vaury C, Jensen S, Kalmykova A. De novo piRNA cluster formation in the Drosophila germ line triggered by transgenes containing a transcribed transposon fragment. Nucleic Acids Res. 2013:41(11):5757-68.

97. Kofler R, Senti K-A, Nolte V, Tobler R, Schlötterer C. Molecular dissection of a natural transposable element invasion. Genome Res. 2018;28(6):824-35.

98. Rozhkov NV, Schostak NG, Zelentsova ES, Yushenova IA, Zatsepina OG, Evgen'ev MB. Evolution and dynamics of small RNA response to a retroelement invasion in Drosophila. Mol Biol Evol. 2013;30(2):397-408.

99. Lu J, Clark AG. Population dynamics of PIWl-interacting RNAs (piRNAs) and their targets in Drosophila. Genome Res. 2010;20(2):212-27.

100. Rahman R, Chirn GW, Kanodia A, Sytnikova YA, Brembs B, Bergman CM, Lau NC. Unique transposon landscapes are pervasive across Drosophila melanogaster genomes. Nucleic Acids Res. 2015;43(22):10655-72.

101. Kelleher ES, Barbash DA. Analysis of piRNA-mediated silencing of active TEs in Drosophila melanogaster suggests limits on the evolution of host genome defense. Mol Biol Evol. 2013;30(8):1816-29.

102. Kelleher ES, Azevedo RBR, Zheng Y. The evolution of small-RNA-mediated silencing of an invading transposable element. Genome Biol Evol. 2018; 10(11):3038-57.

103. Kofler R. Dynamics of transposable element invasions with piRNA clusters. Mol Biol Evol. 2019;36(7):1457-72.

104. Lerat E, Fablet M, Modolo L, Lopez-Maestre H, Vieira C. TEtools facilitates big data expression analysis of transposable elements and reveals an antagonism between their activity and that of piRNA genes. Nucleic Acids Res. 2017:45(4):e17.

105. Obbard DJ, Gordon KH, Buck AH, Jiggins FM. The evolution of RNAi as a defence against viruses and transposable elements. Philos T R Soc B. 2009; 364(1513):99-115.

106. Lee YC, Langley CH. Long-term and short-term evolutionary impacts of transposable elements on Drosophila. Genetics. 2012;192(4):1411-32.

107. Blumenstiel JP, Erwin AA, Hemmer LW. What drives positive selection in the Drosophila piRNA machinery? The genomic autoimmunity hypothesis. Yale J Biol Med. 2016:89(4):499-512.

108. Mackay TFC, Richards S, Stone EA, Barbadilla A, Ayroles JF, Zhu D, Casillas S, Han Y, Magwire MM, Cridland JM, et al. The Drosophila melanogaster genetic reference panel. Nature. 2012;482(7384):173-8.

109. Huang W, Massouras A, Inoue Y, Peiffer J, Ramia M, Tarone AM, Turlapati L, Zichner T, Zhu D, Lyman RF, et al. Natural variation in genome architecture 
among 205 Drosophila melanogaster genetic reference panel lines. Genome Res. 2014;24(7):1193-208.

110. Grenier JK, Arguello JR, Moreira MC, Gottipati S, Mohammed J, Hackett SR, Boughton R, Greenberg AJ, Clark AG. Global Diversity Lines-A five-continent reference panel of sequenced Drosophila melanogaster strains. G3 (Bethesda). 2015;5(4):593-603.

111. Zhuang J, Wang J, Theurkauf W, Weng Z. TEMP: a computational method for analyzing transposable element polymorphism in populations. Nucleic Acids Res. 2014:42(11):6826-38.

112. Cridland JM, Macdonald SJ, Long AD, Thornton KR. Abundance and distribution of transposable elements in two Drosophila QTL mapping resources. Mol Biol Evol. 2013;30(10):2311-27.

113. Adrion JR, Song MJ, Schrider DR, Hahn MW, Schaack S. Genome-wide estimates of transposable element insertion and deletion rates in Drosophila melanogaster. Genome Biol Evol. 2017;9(5):1329-40.

114. Duan Y, Dou S, Luo S, Zhang H, Lu J. Adaptation of A-to-I RNA editing in Drosophila. PLoS Genet. 2017;13(3):e1006648.

115. Lee YC. The role of piRNA-mediated epigenetic silencing in the population dynamics of transposable elements in Drosophila melanogaster. PLoS Genet. 2015;11(6):e1005269.

116. Tajima F. Statistical method for testing the neutral mutation hypothesis by DNA polymorphism. Genetics. 1989;123(3):585-95.

117. Fay JC, Wu C-I. Hitchhiking under positive Darwinian selection. Genetics. 2000;155(3):1405-13.

118. Nielsen R, Williamson S, Kim Y, Hubisz MJ, Clark AG, Bustamante C. Genomic scans for selective sweeps using SNP data. Genome Res. 2005;15(11):1566.

119. Kim Y, Stephan W. Detecting a local signature of genetic hitchhiking along a recombining chromosome. Genetics. 2002;160(2):765-77.

120. Pavlidis P, Jensen JD, Stephan W. Searching for footprints of positive selection in whole-genome SNP data from nonequilibrium populations. Genetics. 2010;185(3):907

121. Pavlidis P, Zivkovic D, Stamatakis A, Alachiotis N. SweeD: likelihood-based detection of selective sweeps in thousands of genomes. Mol Biol Evol. 2013; 30(9):2224-34.

122. Adams MD, Celniker SE, Holt RA, Evans CA, Gocayne JD, Amanatides PG, Scherer SE, Li PW, Hoskins RA, Galle RF, et al. The genome sequence of Drosophila melanogaster. Science. 2000;287(5461):2185-95.

123. Bao W, Kojima KK, Kohany O. Repbase update, a database of repetitive elements in eukaryotic genomes. Mob DNA. 2015;6(1):11.

124. Kent WJ. BLAT-the BLAST-like alignment tool. Genome Res. 2002;12(4): 656-64.

125. Kauer M, Zangerl B, Dieringer D, Schlötterer C. Chromosomal patterns of microsatellite variability contrast sharply in African and non-African populations of Drosophila melanogaster. Genetics. 2002;160(1):247.

126. Glinka S, Ometto L, Mousset S, Stephan W, De Lorenzo D. Demography and natural selection have shaped genetic variation in Drosophila melanogaster: a multi-locus approach. Genetics. 2003;165(3):1269.

127. Haddrill PR, Thornton KR, Charlesworth B, Andolfatto P. Multilocus patterns of nucleotide variability and the demographic and selection history of Drosophila melanogaster populations. Genome Res. 2005;15(6):790-9.

128. Lack JB, Lange JD, Tang AD, Corbett-Detig RB, Pool JE. A thousand fly genomes: an expanded Drosophila genome nexus. Mol Biol Evol. 2016; 33(12):3308-13.

129. Lack JB, Cardeno CM, Crepeau MW, Taylor W, Corbett-Detig RB, Stevens KA, Langley $\mathrm{CH}$, Pool JE. The Drosophila genome Nexus: a population genomic resource of 623 Drosophila melanogaster genomes, including 197 from a single ancestral range population. Genetics. 2015;199(4):1229.

130. Ometto L, Glinka S, De Lorenzo D, Stephan W. Inferring the effects of demography and selection on Drosophila melanogaster populations from a chromosome-wide scan of DNA variation. Mol Biol Evol. 2005;22(10):2119-30.

131. Kapopoulou A, Pfeifer SP, Jensen JD, Laurent S. The demographic history of African Drosophila melanogaster. Genome Biol Evol. 2018;10(9):2338-42.

132. Li H, Stephan W. Inferring the demographic history and rate of adaptive substitution in Drosophila. PLoS Genet. 2006;2(10):e166.

133. Charlesworth B. Effective population size and patterns of molecular evolution and variation. Nat Rev Genet. 2009;10(3):195-205.

134. Wen J, Mohammed J, Bortolamiol-Becet D, Tsai H, Robine N, Westholm JO, Ladewig E, Dai Q, Okamura K, Flynt AS, et al. Diversity of miRNAs, siRNAs, and piRNAs across 25 Drosophila cell lines. Genome Res. 2014;24(7):1236-50.

135. Yin $\mathrm{H}$, Lin $\mathrm{H}$. An epigenetic activation role of Piwi and a Piwi-associated piRNA in Drosophila melanogaster. Nature. 2007;450(7167):304-8.
136. Clark JB, Kidwell MG. A phylogenetic perspective on P transposable element evolution in Drosophila. Proc Natl Acad Sci U S A. 1997;94(21):11428-33.

137. Li C, Vagin W, Lee S, Xu J, Ma S, Xi H, Seitz H, Horwich MD, Syrzycka M, Honda BM, et al. Collapse of germline piRNAs in the absence of Argonaute3 reveals somatic piRNAs in flies. Cell. 2009;137(3):509-21.

138. Bergman CM, Bensasson D. Recent LTR retrotransposon insertion contrasts with waves of non-LTR insertion since speciation in Drosophila melanogaster. Proc Natl Acad Sci U S A. 2007;104(27):11340-5.

139. Dolgin ES, Charlesworth $B$. The effects of recombination rate on the distribution and abundance of transposable elements. Genetics. 2008;178(4):2169.

140. Charlesworth B, Sniegowski P, Stephan W. The evolutionary dynamics of repetitive DNA in eukaryotes. Nature. 1994;371:215.

141. Le Thomas A, Rogers AK, Webster A, Marinov GK, Liao SE, Perkins EM, Hur JK, Aravin AA, Toth KF. Piwi induces piRNA-guided transcriptional silencing and establishment of a repressive chromatin state. Genes Dev. 2013;27(4):390-9.

142. Sienski G, Donertas D, Brennecke J. Transcriptional silencing of transposons by Piwi and maelstrom and its impact on chromatin state and gene expression. Cell. 2012;151(5):964-80.

143. Senti KA, Jurczak D, Sachidanandam R, Brennecke J. piRNA-guided slicing of transposon transcripts enforces their transcriptional silencing via specifying the nuclear piRNA repertoire. Genes Dev. 2015;29(16):1747-62.

144. Le Thomas A, Stuwe E, Li S, Du J, Marinov G, Rozhkov N, Chen Y-CA, Luo Y, Sachidanandam R, Toth KF. Transgenerationally inherited piRNAs trigger piRNA biogenesis by changing the chromatin of piRNA clusters and inducing precursor processing. Genes Dev. 2014;28(15):1667-80.

145. van Houte S, Buckling A, Westra ER. Evolutionary ecology of prokaryotic immune mechanisms. Microbiol Mol Biol Rev. 2016;80(3):745-63.

146. Barron MG, Fiston-Lavier AS, Petrov DA, Gonzalez J. Population genomics of transposable elements in Drosophila. Annu Rev Genet. 2014;48:561-81.

147. Bolger AM, Lohse M, Usadel B. Trimmomatic: a flexible trimmer for Illumina sequence data. Bioinformatics. 2014:30(15):2114-2120.

148. Li H, Durbin R. Fast and accurate short read alignment with burrowsWheeler transform. Bioinformatics. 2009;25(14):1754-60.

149. Edgar RC. Search and clustering orders of magnitude faster than BLAST. Bioinformatics. 2010;26(19):2460-1.

150. Kruskal JB, Wish M. Multidimensional Scaling. In: Sage University paper series on quantitative applications in the social sciences. Newbury Park: Sage Publications; 1978. p. 7-11.

151. Danecek P, Auton A, Abecasis G, Albers CA, Banks E, DePristo MA, Handsaker RE, Lunter G, Marth GT, Sherry ST. The variant call format and VCFtools. Bioinformatics. 2011;27(15):2156-8.

152. Cingolani P, Platts A, Wang LL, Coon M, Nguyen T, Wang L, Land SJ, Lu X, Ruden DM. A program for annotating and predicting the effects of single nucleotide polymorphisms, SnpEff: SNPs in the genome of Drosophila melanogaster strain W1118; iso-2; iso-3. Fly. 2012;6(2):80-92.

153. Rosenbloom KR, Armstrong J, Barber GP, Casper J, Clawson H, Diekhans M, Dreszer TR, Fujita PA, Guruvadoo L, Haeussler M. The UCSC genome browser database: 2015 update. Nucleic Acids Res. 2015:43(D1):D670-81.

154. Barrett JC. Haploview: Visualization and analysis of SNP genotype data. Cold Spring Harb Protoc. 2009;2009(10):pdb. ip71.

155. Kim D, Pertea G, Trapnell C, Pimentel H, Kelley R, Salzberg SL. TopHat2: accurate alignment of transcriptomes in the presence of insertions, deletions and gene fusions. Genome Biol. 2013;14(4):R36.

156. Anders S, Pyl PT, Huber W. HTSeq-A Python framework to work with highthroughput sequencing data. Bioinformatics. 2014;31(2):166-169.

157. Dobin A, Davis CA, Schlesinger F, Drenkow J, Zaleski C, Jha S, Batut $P$, Chaisson M, Gingeras TR. STAR: ultrafast universal RNA-seq aligner. Bioinformatics. 2013;29(1):15-21.

158. Martin M. Cutadapt removes adapter sequences from high-throughput sequencing reads. EMBnet J. 2011;17(1):10-2.

159. Langmead B, Salzberg SL. Fast gapped-read alignment with bowtie 2. Nat Methods. 2012;9(4):357-9.

160. Antoniewski C. Computing siRNA and piRNA overlap signatures. In: Werner A, editor. Animal Endo-SiRNAs: Methods and Protocols. New York: Springer New York; 2014. p. 135-46.

161. Quinlan AR, Hall IM. BEDTools: a flexible suite of utilities for comparing genomic features. Bioinformatics. 2010;26(6):841-2.

\section{Publisher's Note}

Springer Nature remains neutral with regard to jurisdictional claims in published maps and institutional affiliations. 